\title{
Resource allocation for the multiple-access relay channels and OFDMA
}

\author{
Stephan Schedler ${ }^{*}$ and Volker Kühn
}

\begin{abstract}
Within this article, the resource allocation problem for the multiple-access relay channel with orthogonal channel access is analysed. We propose a centralised optimisation framework that maximises the achievable sum rate of the network and that can be applied to a wide range of scenarios. We analyse different approaches to tackle the problem with individual transmit power constraints such as relaxing of constraints and splitting of the joint problem into a carrier allocation and individual power allocations. The carrier allocation deals with the exclusive assignment of subcarriers to different users, and the power allocation addresses the distribution of available power over assigned subcarriers. Several approaches are proposed for amplify and forward, decode and forward, and compress and forward strategies, and the performance as well as computational effort is evaluated by simulations. The obtained results suggest that the proposed algorithms can perform close to optimal.
\end{abstract}

Keywords: MARC; Relay; OFDMA; Carrier allocation; Power allocation; Dual optimisation; Sum rate; Amplify and forward (AF); Decode and forward (DF); Compress and forward (CF)

\section{Introduction}

Recently it was shown that the throughput in a network can be significantly increased using multiple-input multiple-output or advanced error correction techniques. However, this might not be sufficient to provide a required quality of service if there are long distances between the users and the base station. It is well known that relays can improve communication between the base station and mobile nodes in such a situation. Especially if a user is close to the cell edge, the network can benefit from assisting relay nodes. Relay nodes can enhance throughput, save power, increase the coverage area or fill some coverage black spots without the need for a wired backhaul connection.

Yet, relay nodes introduce additional complexity to resource allocation problems. The use of available bandwidth and transmit power has to be optimised to utilise relays efficiently. The optimal choice of resource allocations depends on several parameters, such as the considered channel model (e.g. fading), the applied relaying protocol or the actual topology of the network.

*Correspondence: stephan.schedler@uni-rostock.de Institute of Communications Engineering, University of Rostock, Richard-Wagner-Str 31, Warnemünde, Rostock 18119, Germany
Furthermore, a sophisticated protocol is required for message forwarding or relay selection in case of multiple relay nodes. Hence, many non-trivial optimisation problems are connected to relay networks. Within this work, we will analyse some of these for a multipleaccess relay channel (MARC) assuming a half-duplex constraint, orthogonal channel access and individual power constraints.

\subsection{Related work}

The general relay channel model was introduced in 1971 by van der Meulen in [1,2]. In 1979, Cover and Gamal analysed 'Capacity theorems for the relay channel' [3] in great detail. The same work has also introduced two fundamental coding strategies, nowadays well known as decode and forward (DF) and compress and forward (CF).

Research in the area of relay networks attracted much attention in the last years due to improved capabilities in the field of computer science. Today, even mobile devices can provide sufficient computing power for relaying. It is therefore reasonable that relaying has been considered during the standardisation processes of actual mobile radio standards such as LTE-Advanced. Furthermore, much work has been spent to determine capacity

\section{至 Springer}

(c) 2013 Schedler and Kühn; licensee Springer. This is an Open Access article distributed under the terms of the Creative

Commons Attribution License (http://creativecommons.org/licenses/by/2.0), which permits unrestricted use, distribution, and reproduction in any medium, provided the original work is properly cited. 
bounds and rate regions for relay networks. The singleuser Gaussian relay channel as well as the MARC has been analysed, for example, in [4-9] with respect to (w.r.t) outage probabilities or achievable transmission rates and a wide range of relay protocols.

In order to reach the obtained theoretical bounds, several optimisation strategies have been derived for solving resource allocation problems. Starting with a single-user point-to-point orthogonal frequency division multiplexing (OFDM) system, the optimal power allocation is obtained by the well-known water-filling solution [10]. The solution for multiple users in an orthogonal frequency division multiple access (OFDMA) system is more involved due to the additional carrier allocation (CA) problem. OFDMA uplink and downlink have been considered by several authors, and many solutions have been proposed [11-18]. In general, there are three different approaches to solve such a mixed integer programming problem: The exhaustive search checks all possible subcarrier assignments $[16,18]$, relaxing of constraints transforms integers into real valued variables $[11,14]$ and dual decomposition searches for the optimal CA in the dual domain [16,17]. Yin and Lui [13] showed that the subcarrier allocation for the OFDMA downlink can be solved efficiently by the Hungarian algorithm.

A different power allocation problem arises for a relayassisted single-user OFDM system [19-30]. An iterative algorithm has been proposed by HammerstrÃüm and Wittneben in [21,22] for the case of amplify and forward (AF). Low-complexity algorithms for DF strategies were proposed by Zhang and Lau [23] and Vandendorpe et al. $[24,25,27]$. Wang et al. [20], Boostanimehr et al. [28], Shi and Zhao [29] and Hsu et al. [30] considered subcarrier pairing to exploit spatial diversity.

The resource allocation problem considered within this work combines both problems. Power and subcarrier allocation will be analysed in an uplink OFDMA system that is assisted by a relay. $\mathrm{Ng}$ and $\mathrm{Yu}$ [31] and Jitvanichphaibool et al. [32] considered the relay selection and power allocation problem for AF and DF strategies. They combine a dual decomposition approach with an exhaustive search for the relay selection. However, an exhaustive search over all possible carrier allocations is only feasible for few subcarriers or if the number of subcarriers per user is fixed. Furthermore, dual decomposition cannot be applied to our problem since our problem is not separable in the subcarriers. If the subcarrier assignment is changed, power constraints of multiple nodes are affected. El Soussi et al. [33] considered a power allocation (PA) problem for the downlink where subcarriers might be shared between source and relay nodes, but their results suggest that at most one user should be relayed per subcarrier. Furthermore, the solution of the dual optimisation in that work is not applicable to our scenario as a sum power constraint is considered.

\subsection{Contributions}

Within this article, the general resource allocation problem is analysed for the half-duplex MARC with orthogonal channel access and individual power constraints at all nodes. Contrary to some publications referenced in the previous section, we will look at the joint problem with a single relay and multiple users. However, we will not neglect the direct path, and we will consider individual power constraints for all nodes. We formulate the nonconcave problem for the MARC with OFDMA and give a survey of different centralised methods to tackle the maximisation of the achievable sum rate for a wide range of relaying strategies. The considered approaches are as follows:

- Relaxing of constraints and decomposition into individual subproblems

- Solving the Lagrangian dual problem for different relaying strategies

- A simple gradient method that achieves excellent results even if the global problem is not concave

- A modification of the Hungarian algorithm to solve the CA problem in polynomial time

- An improved greedy CA

In general, most of the considered methods have been applied to different or closely related optimisation problems before. Within this article, we will show how these approaches can be utilised for the MARC with OFDMA. Required modifications, derivations, as well as possible improvements are presented. Furthermore, we provide several simulation results, present a comparison of the considered methods and give a brief analysis of the related computational effort.

The rest of this article is structured as follows: First, the analysed system model, basic assumptions, relay protocols and a formal problem description are introduced in 2. Afterwards, optimal and near-optimal algorithms to solve the global resource allocation problem (3) as well as carrier allocation (4) and power allocation (5) subproblems are derived. Finally, 6 presents numerical results of simulations and compares the proposed algorithms.

\section{Problem description}

This section will introduce the network, channel models, as well as relay strategies that are considered for the optimisation of the achievable sum rates. Afterwards, a mathematical description of the general resource allocation problem will be introduced. 


\subsection{System model}

Within this work, a wireless MARC with mobile nodes is considered. In general, there is a set of $K$ source nodes $\mathcal{S}_{k}$ with $k \in \mathbb{K}=\{0, \ldots, K-1\}$, a single destination $\mathcal{D}$ and a single relay node $\mathcal{R}$. The source nodes $\mathcal{S}_{k}$ transmit independent messages to the destination $\mathcal{D}$, and the relay node $\mathcal{R}$ assists in the transmission, improving the achievable rates. The position of any node $\mathcal{A}$ in the $x y$-plane is given by its coordinates $x^{(\mathcal{A})}$ and $y^{(\mathcal{A})}$. The Euclidean distance between any two nodes $\mathcal{A}$ and $\mathcal{B}$ is then denoted $d_{(\mathcal{A B})}$. A simple example with three source nodes is depicted in Figure 1.

Since communication is assumed to be wireless, a multiple-access scheme has to be chosen such that there is no interference between the data streams of different nodes. Within this work, a half-duplex scheme is assumed, i.e. a node cannot transmit and receive simultaneously. The considered transmission scheme will be a combination of a time division multiple access and an OFDMA scheme.

In the first time slot, the source nodes $\mathcal{S}_{k}$ are allowed to send their information while relay $\mathcal{R}$ and destination $\mathcal{D}$ receive messages. Afterwards, the relay can forward previously received messages in a second time slot with the same length. Finally, destination $\mathcal{D}$ can combine obtained information from both time slots.

Interference between the data streams of different users is avoided by separation in frequency domain. OFDMA splits the available bandwidth into a set of $N$ orthogonal subcarriers $\mathbb{N}=\{0, \ldots, N-1\}$. These subcarriers are assigned exclusively to the source nodes, where $\mathbb{N}_{k} \subset \mathbb{N}$ is a subset of the exclusively assigned subcarriers $\left(\mathbb{N}_{i} \cap \mathbb{N}_{j}=\emptyset \forall i \neq j\right)$ to source node $\mathcal{S}_{k}$.

The cardinality of this set is $\left|\mathbb{N}_{k}\right|$. The subcarrier assignments $\mathbb{N}_{k}$ are valid for source nodes $\mathcal{S}_{k}$ as well as for relay $\mathcal{R}$. Subcarrier pairing as proposed in $[20,28]$ will not be considered.

In general, a frequency-selective channel of bandwidth $B$ is assumed. Due to the application of OFDMA, the links between nodes $\mathcal{A}$ and $\mathcal{B}$ on a single subcarrier $n$ can be characterised by a scalar factor $H_{n}^{(\mathcal{A B})}$. These channel coefficients are assumed to be i.i.d. They are circular

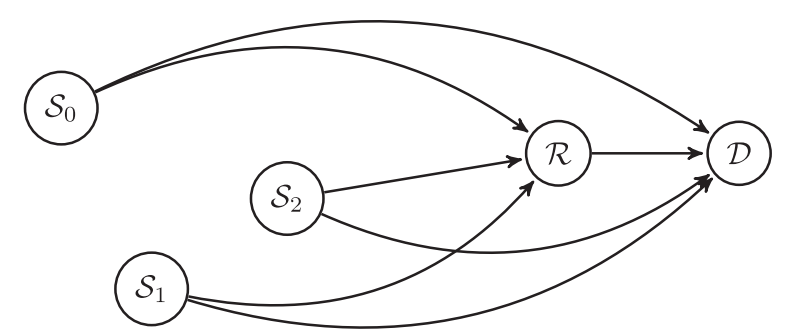

Figure 1 Example of a three-user MARC. symmetric normal distributed $H_{n}^{(\mathcal{A B})} \sim \mathcal{C N}\left(0, d_{(\mathcal{A B})}^{-\alpha}\right)$, and their variance has been chosen such that the expectation of the absolute value $E\left\{\left|H_{n}^{(\mathcal{A B})}\right|\right\}$ becomes path loss factor $\sqrt{d_{(\mathcal{A B})}^{-\alpha}}$. Furthermore, we assume a slow-fading environment, i.e. the channel coefficients are assumed to be constant over time.

Each transmission is also disturbed by additive white Gaussian noise of variance $\sigma_{\mathrm{N}}^{2}$ leading to the signal-tonoise ratio (SNR) of subcarrier $n$ at receiver $\mathcal{B}$

$$
\gamma_{n}^{(\mathcal{A B})}=\frac{p_{n}^{(\mathcal{A})}\left|H_{n}^{(\mathcal{A B})}\right|^{2}}{\sigma_{\mathrm{N}}^{2}} .
$$

In (1), $p_{n}^{(\mathcal{A})}$ is the power node $\mathcal{A}$ spends for transmission on the $n$th subcarrier. For the sake of clarity, the channelto-noise ratio of the $n$th subcarrier will be denoted by $\lambda_{n}^{(\mathcal{A B})}=\frac{\left|H_{n}^{(\mathcal{A B})}\right|^{2}}{\sigma_{\mathrm{N}}^{2}}$.

According to the definition in [34, Theorem 17], the channel capacity for a point-to-point communication on a single subcarrier $n$ between two nodes $\mathcal{A}$ and $\mathcal{B}$ is given by

$$
C_{n}^{(\mathcal{A B})}=\frac{B}{N} \log _{2}\left(1+\gamma_{n}^{(\mathcal{A B})}\right) .
$$

The power that transmitter $\mathcal{A}$ spends to send a message over the $n$th subcarrier is given by $p_{n}^{(\mathcal{A})} \geq 0$. The vector containing the complete power allocation at node $\mathcal{A}$ is denoted by $\boldsymbol{p}^{(\mathcal{A})}=\left[p_{0}^{(\mathcal{A})}, \ldots, p_{N-1}^{(\mathcal{A})}\right]^{T}$.

Each node is equipped with an individual power supply. For fixed time slot lengths, the available transmit power per time slot is thus limited by individual power constraints

$$
\sum_{n \in \mathbb{N}} p_{n}^{(\mathcal{A})} \leq P_{\max } \quad \forall \mathcal{A} \in\left\{\mathcal{R}, \mathcal{S}_{0}, \ldots, \mathcal{S}_{K-1}\right\} .
$$

Finally, we have to choose the constraint $P_{\max }$ such that the average energy consumption is independent of $N$ and $K$. This can be achieved by solving the average SNR of a single subcarrier

$$
E\left\{\gamma_{n}^{\left(\mathcal{S}_{k} \mathcal{D}\right)}\right\}=\frac{d_{\left(\mathcal{S}_{k} \mathcal{D}\right)}^{-\alpha} P_{\max }}{\sigma_{\mathrm{N}}^{2}}
$$

for $P_{\max }$. In (4), the term $\frac{P_{\max }}{N / K}$ is the available average power per subcarrier and user.

This normalisation is necessary to ensure a fair comparison of simulation results for different $N$.

\subsection{Relay protocols}

In general, the relay assists the source nodes $\mathcal{S}_{k}$ in transmitting messages to destination $\mathcal{D}$. Using a relay might be reasonable if the direct link is poor due to a large path loss or shadowing. Here, a relay can improve the communication by forwarding received signals from source nodes towards the destination. In the considered MARC, 
a single relay node $\mathcal{R}$ assists multiple source nodes $\mathcal{S}_{k}$ in the transmission of information over $N$ orthogonal subcarriers. However, the actual throughput of the MARC depends on the protocol that is used at the relay node.

\subsubsection{Amplify and forward}

Amplify and Forward is a simple protocol, where the relay node $\mathcal{R}$ just rescales the received signal before retransmitting it to the destination node $\mathcal{D}$. Afterwards, node $\mathcal{D}$ performs maximum ratio combining of received messages from the first and second time slot.

The achievable transmission rate for the AF protocol is given by [5]

$$
\begin{aligned}
r_{n, k}^{\mathrm{AF}}= & \frac{1}{2} \frac{B}{N} \log _{2}\left(1+p_{n}^{\left(\mathcal{S}_{k}\right)} \lambda_{n}^{\left(\mathcal{S}_{k} \mathcal{D}\right)}\right. \\
& \left.+\frac{p_{n}^{\left(\mathcal{S}_{k}\right)} \lambda_{n}^{\left(\mathcal{S}_{k} \mathcal{R}\right)} p_{n}^{(\mathcal{R})} \lambda_{n}^{(\mathcal{R D})}}{p_{n}^{\left(\mathcal{S}_{k}\right)} \lambda_{n}^{\left(\mathcal{S}_{k} \mathcal{R}\right)}+p_{n}^{(\mathcal{R})} \lambda_{n}^{(\mathcal{R D})}+1}\right),
\end{aligned}
$$

where factor $1 / 2$ is caused by the half-duplex constraint.

\subsubsection{Decode and forward}

Another common strategy is to decode and forward received messages at the relay. After relay $\mathcal{R}$ successfully decoded the received message, the information is re-encoded with the same code and forwarded to the destination in the second time slot. Therefore, relay $\mathcal{R}$ retransmits exactly the same code word as the source node.

If $\mathcal{R}$ is constrained to decode received messages successfully, the scheme will be denoted as non-adaptive decode and forward (nDF). If $\mathcal{R}$ would not be able to decode without errors, the transmission rate has to be reduced such that successful transmission over the $\mathcal{S} \rightarrow \mathcal{R}$ path is possible. The achievable transmission rate on a subcarrier is therefore limited by the SNR at relay $\mathcal{R}$ and by the SNR at destination $\mathcal{D}$. The rate $r_{n, k}^{\mathrm{nDF}}$ of the non-adaptive $\mathrm{DF}$ strategy is given by [5, Equation (15)]

$$
\begin{aligned}
r_{n, k}^{\mathcal{D}} & =\frac{1}{2} \frac{B}{N} \log _{2}\left(1+p_{n}^{\left(\mathcal{S}_{k}\right)} \lambda_{n}^{\left(\mathcal{S}_{k} \mathcal{D}\right)}+p_{n}^{(\mathcal{R})} \lambda_{n}^{(\mathcal{R} \mathcal{D})}\right) \\
r_{n, k}^{\mathcal{R}} & =\frac{1}{2} \frac{B}{N} \log _{2}\left(1+p_{n}^{\left(\mathcal{S}_{k}\right)} \lambda_{n}^{\left(\mathcal{S}_{k} \mathcal{R}\right)}\right) \\
r_{n, k}^{\mathrm{nDF}} & =\min \left\{r_{n, k}^{\mathcal{D}}, r_{n, k}^{\mathcal{R}}\right\} .
\end{aligned}
$$

The rate $r_{n, k}^{\mathcal{R}}$ corresponds to the maximum rate at which relay $\mathcal{R}$ can decode the source message successfully, while the term $r_{n, k}^{\mathcal{D}}$ represents the rate at which destination $\mathcal{D}$ can reliably decode.

In contrast to that, an adaptive decode and forward (aDF) might switch between non-aDF and direct transmission depending on the actual channel states. If the relay is not able to decode successfully on the $n$th subcarrier, a transmission over the direct path is the better choice and the relay will be turned off for that subcarrier. The transmission rate $r_{n, k}^{\mathrm{aDF}}$ can be formulated as a combination of $\mathrm{nDF}$ and direct transmission:

$$
\begin{aligned}
r_{n, k}^{\mathrm{Dir}} & =\frac{1}{2} \frac{B}{N} \log _{2}\left(1+p_{n}^{\left(\mathcal{S}_{k}\right)} \lambda_{n}^{\left(\mathcal{S}_{k} \mathcal{D}\right)}\right) \\
r_{n, k}^{\mathrm{aDF}} & =\max \left\{r_{n, k}^{\mathrm{nDF}}, r_{n, k}^{\mathrm{Dir}}\right\} .
\end{aligned}
$$

\subsubsection{Compress and forward}

The AF strategy amplifies not only the signal energy but also the noise received at the relay. Thus, the destination node has to deal with its own noise and with the amplified noise from the $(\mathcal{S}) \rightarrow \mathcal{R}$ link. Contrary, the DF strategy removes the influence of noise from the $(\mathcal{S}) \rightarrow \mathcal{R}$ path by decoding messages at the relay node. However, successful decoding at the relay $\mathcal{R}$ has to be ensured for that strategy.

A third relay protocol known as CF was proposed by Cover and Gamal in [3]. The CF strategy can be considered as a trade-off between the previously discussed strategies. The main idea is to compress the observed message at relay $\mathcal{R}$ before forwarding. Relay $\mathcal{R}$ does not decode the received messages, but it quantises (i.e. compresses) the received observations and re-encodes and transmits them to destination $\mathcal{D}$. Hence, useful information, as well as noise from the $\mathcal{S} \rightarrow \mathcal{R}$ path, will be forwarded to the destination. The advantage over the AF protocol lies in the property that even if there is some additional distortion $Z_{w}$ due to quantisation, the noise of the $\mathcal{S} \rightarrow \mathcal{R}$ path will not be amplified. In case of CF, the additional distortion is controlled by the quantisation, and it is chosen such that there is still no further loss of information on the second hop.

The achievable transmission rate for CF in a wireless, half-duplex, Gaussian relay channel has been derived by Høst-Madsen and Zhang for the non-orthogonal case in [7, Equation (15)]. The solution can easily be adjusted to the considered relay network within this work. It is given by

$$
r_{n, k}^{\mathrm{CF}}=\frac{1}{2} \frac{B}{N} \log _{2}\left(1+p_{n}^{\left(\mathcal{S}_{k}\right)} \lambda_{n}^{\left(\mathcal{S}_{k} \mathcal{D}\right)}+\frac{p_{n}^{\left(\mathcal{S}_{k}\right)} \lambda_{n}^{\left(\mathcal{S}_{k} \mathcal{D}\right)}}{1+\sigma_{w}^{2}}\right)
$$

where $\sigma_{w}^{2}$ is the variance of the compression noise $Z_{w} \sim \mathcal{C N}\left(0, \sigma_{w}^{2}\right)$.

The derivation of the optimal choice of variance $\sigma_{w}^{2}$ is not shown here but can be found in $[7,8]$ :

$$
\sigma_{w}^{2}=\frac{p_{n}^{\left(\mathcal{S}_{k}\right)} \lambda_{n}^{\left(\mathcal{S}_{k} \mathcal{D}\right)}+p_{n}^{\left(\mathcal{S}_{k}\right)} \lambda_{n}^{\left(\mathcal{S}_{k} \mathcal{R}\right)}+1}{p_{n}^{(\mathcal{R})} \lambda_{n}^{(\mathcal{R D})}\left(p_{n}^{\left(\mathcal{S}_{k}\right)} \lambda_{n}^{\left(\mathcal{S}_{k} \mathcal{R}\right)}+1\right)}
$$

\subsection{General resource allocation problem}

Next, we will define the sum rate $R$ of the whole network. It is given by the summation of rates $r_{n, k}$ over all users 
$k \in \mathbb{K}$ and all subcarriers $n \in \mathbb{N}_{k}$ that have been assigned to that user:

$$
R=\sum_{k \in \mathbb{K}} \sum_{n \in \mathbb{N}_{k}} r_{n, k} .
$$

The specific rates $r_{n, k}$ that source node $(\mathcal{S})_{k}$ can achieve on the $n$th subcarrier depend on the applied relaying strategy and have been defined in Section 2.2. Combining the above definitions, it is possible to formulate the general resource allocation problem that will be analysed within this work. In general, we look for the CA and PA that maximise the achievable sum rate $R$ :

$$
\begin{aligned}
& \left\{\mathbb{N}_{k}^{*}, \boldsymbol{p}^{*\left(\mathcal{S}_{k}\right)}, \boldsymbol{p}^{*(\mathcal{R})}\right\}_{k=0}^{K-1}=\underset{\left\{\mathbb{N}_{k}, \boldsymbol{p}^{\left(\mathcal{S}_{k}\right)}, \boldsymbol{p}^{(\mathcal{R})}\right\}_{k=0}^{K-1}}{\operatorname{argmax}} R \\
& \text { s.t. } \quad p_{n}^{\left(\mathcal{S}_{k}\right)} \geq 0 \quad \forall n \in \mathbb{N}, k \in \mathbb{K} \\
& p_{n}^{(\mathcal{R})} \geq 0 \quad \forall n \in \mathbb{N} \\
& \sum_{n \in \mathbb{N}_{k}} p_{n}^{\left(\mathcal{S}_{k}\right)} \leq P_{\max } \quad \forall k \in \mathbb{K} \\
& \sum_{n \in \mathbb{N}} p_{n}^{(\mathcal{R})} \leq P_{\max } \\
& \mathbb{N}_{i} \cap \mathbb{N}_{j}=\emptyset \quad \forall i \neq j \\
& \bigcup_{k \in \mathbb{K}} \mathbb{N}_{k}=\mathbb{N}
\end{aligned}
$$

Since CAs have to be exclusive, this problem is a mixed integer programming problem. As an alternative to the set notation used so far, the subcarrier allocations can also be described by an assignment matrix:

$$
\mathbf{Z}=\left[\begin{array}{ccc}
\mathrm{z}_{0,0} & \cdots & \mathrm{z}_{0, K-1} \\
\vdots & \ddots & \vdots \\
\mathbf{z}_{N-1,0} & \cdots & \mathrm{z}_{N-1, K-1}
\end{array}\right]=\left[\begin{array}{c}
\mathbf{z}_{0} \\
\vdots \\
\mathbf{z}_{N-1}
\end{array}\right]
$$

If the element $\mathrm{z}_{n, k}$ is equal to 1 , subcarrier $n$ is allocated to source node $\mathcal{S}_{k}$. Consequently, it is allocated to some other source node if $\mathrm{z}_{n, k}=0$ :

$$
\mathrm{z}_{n, k}=\left\{\begin{array}{l}
1 \forall n \in \mathbb{N}_{k} \\
0 \forall n \notin \mathbb{N}_{k}
\end{array}\right.
$$

The exclusive subcarrier assignments (19) and (20) can also be expressed by

$$
\begin{array}{ll}
\mathrm{z}_{n, k} \in\{0,1\} & \forall n \in \mathbb{N}, k \in \mathbb{K} \\
\sum_{k \in \mathbb{K}} \mathrm{z}_{n, k}=1 & \forall n \in \mathbb{N} .
\end{array}
$$

The modified notation with variables $z_{n, k}$ is equivalent to the previously defined sets $\mathbb{N}_{k}$ and enables easier explanations for the proposed algorithms.

In order to maximise the sum rate, the resource allocation problem (14) to (20) has to be solved. We consider a slow-fading environment where full channel state information (CSI) is available at a central node, i.e. that node knows the fading coefficients of all links between all nodes. The resource allocation is performed in a centralised way. It is not necessarily part of the transmission scheme, but it manages the allocation of available resources in the network. The additional effort due to signalling to the central node as well as the required protocols to share side information is neglected within this work. We are well aware that this is a critical point as the actual throughput of the system might be much lower than the achievable sum rate $R$, but overhead analysis and partial CSI are beyond the scope of this work. We refer interested readers to [35] where a distributed resource allocation scheme with partial CSI is proposed that has a communication overhead of at most $K \cdot \log _{2} N$ bits. It is shown in [35] that the reduction in throughput due to partial CSI is moderate if diversity can be exploited.

\section{Joint allocation}

The next sections will introduce a number of algorithms to solve the resource allocation problem. First, the joint allocation (JA) problem will be considered. Afterwards, CA and PA problems are analysed separately in subsequent sections. In general, the description of algorithms will not be restricted to a specific relay protocol. If there are some additional aspects for a particular relay protocol, they will be considered after the general description.

\subsection{Exhaustive search}

The exhaustive will be used as a reference and an upper bound for the achievable sum rate. The main idea is to check all possible subcarrier assignments $\mathbb{N}_{k}$ of all source nodes $\mathcal{S}_{k}$. As the sum rate $R$ depends on the CA as well as the PA, the optimal choice of sets $\mathbb{N}_{k}$ can only be found if the PA problem is solved for each possible CA. However, since sum rate $R$ is not concave w.r.t the PA, finding the global optimum is hardly feasible. For the analysis, the Optimization Toolbox V6.0 of Matlab R2011a has been run 50 times with different initial values per channel realisation to determine the PA. After checking all usersubcarrier combinations, the result with the best sum rate $R$ will be chosen.

On the one hand, this algorithm might be able to find the optimal subcarrier assignments, but on the other hand, the computational effort $\mathcal{O}\left(K^{N}\right)$ to solve the integer problem is infeasible for practical applications. For a small problem with $K=2$ users and $N=4$ subcarriers, 
the computation is done quickly. For $N=256$ subcarriers, however, the exhaustive cannot be done in reasonable time as there are $2^{256} \approx 10^{77}$ possible subcarrier assignments.

\subsection{Relaxing of constraints}

A common approach to solve integer problems is relaxing their constraints [11,17]. As the combinatorial problem within this work is caused by the exclusive subcarrier allocations, relaxing constraint (23) leads to real valued subcarrier assignments

$$
\mathrm{z}_{n, k} \in[0,1]
$$

Subcarriers are thus no longer assigned exclusively to a single user, but multiple users can share a single carrier. In general, this is incompatible with the definitions made above, but it is reasonable under certain conditions. If there are $M$ OFDM symbols to be transmitted and $M$ is large, source node $\mathcal{S}_{k}$ is allowed to transmit $M \cdot \mathrm{z}_{n, k}$ symbols on subcarrier $n$. The remaining $M \cdot\left(1-\mathrm{z}_{n, k}\right)$ OFDM symbols belong to other source nodes. With this interpretation, the channel access can again be done exclusively, but each subcarrier might be shared over time. In general, time sharing [11] is a reasonable assumption if the channel is constant over a long period of time.

\subsubsection{Gradient descent method}

The relaxed JA problem can be tackled with a modified gradient descent method [36]. Since subcarrier allocations $\mathrm{z}_{n, k}$ are real valued now, objective $R$ is continuously differentiable and partial derivatives can be computed for all variables.

The basic idea of the iterative algorithm can be described by an iterative update:

$$
\boldsymbol{x}[i+1]=\boldsymbol{x}[i]+\vartheta_{x} R_{\partial x}[i],
$$

where $i$ is an iteration counter and vector $x$ is a substitution for the resource allocation variables $\left\{\mathbf{z}_{n}\right\}_{n=0}^{N-1}$, $\left\{\boldsymbol{p}^{\left(\mathcal{S}_{k}\right)}\right\}_{k=0}^{K-1}$ and $\boldsymbol{p}^{(\mathcal{R})}$. The variable $\vartheta_{x}$ is a scalar step size, and $R_{\partial x}$ is an iterative update variable. Next, we have to compute a feasible update direction. The initial value of $R_{\partial x}$ corresponds to the partial derivative

$$
R_{\partial x}[i]=\frac{\partial R[i]}{\partial x} .
$$

As the gradient of sum rate $R$ is always positive for all elements, the updated allocation vectors $x[i+1]$ always violate the individual sum constraints (17), (18) or (24). To stay in a feasible region, the gradient vector has to be projected back to the feasible region. This can be achieved by subtracting the mean values of partial derivatives:

$$
R_{\partial x}[i]=R_{\partial x}[i]-\overline{R_{\partial x}[i]} .
$$

The modified gradient spends more power for elements with a strong gradient and removes some power from elements with low gradients. If this again leads to a negative element $x[i+1]$, it has to be further adjusted such that $\boldsymbol{x}[i+1]$ fulfils the lower bounds (15), 16 and (25):

$$
R_{\partial x}[i]=\max \left\{R_{\partial x}[i],-\frac{x[i]}{\vartheta_{x}}\right\} .
$$

After $R_{\partial x}[i]$ has been computed, the new resource allocations $\boldsymbol{x}[i+1]$ and the corresponding sum rate $R[i+1]$ can be calculated for a given step size $\vartheta_{x}$.

The proposed algorithm will be repeated iteratively until the achieved gain becomes smaller than a constant $\epsilon$. For simulation, $\epsilon=10^{-8}$ will be chosen as stopping condition and the initial allocation elements are given by the average values:

$$
\begin{array}{rlrl}
p_{n}^{\left(\mathcal{S}_{k}\right)}(0) & =\frac{1}{N / K} P_{\max } & & \forall n \in \mathbb{N}, k \in \mathbb{K} \\
p_{n}^{(\mathcal{R})}(0) & =\frac{1}{N} P_{\max } & & \forall n \in \mathbb{N} \\
\mathrm{z}_{n, k}(0)=\frac{1}{K} & & \forall n \in \mathbb{N}, k \in \mathbb{K} .
\end{array}
$$

A summary of the proposed gradient algorithm is shown in Algorithm 1.

For low SNRs, the subcarrier assignments $\mathrm{z}_{n, k}$ converge to integer values $\{0,1\}$ due to the choice of appropriate feasibility updates (28) and (29). If subcarrier allocation $\mathrm{z}_{n, k}$ becomes low or close to 0 for an arbitrary subcarrier, the gradient and, therefore, the value of the corresponding power $p_{n}^{\left(\mathcal{S}_{k}\right)}$ will also become small in the next iteration. Since the gradient of $\mathrm{z}_{n, k}$ again depends on power $p_{n}^{\left(\mathcal{S}_{k}\right)}$, a value that once became low can merely become high again and $\mathrm{z}_{n, k}$ will become equal to 1 or 0 after few iterations.

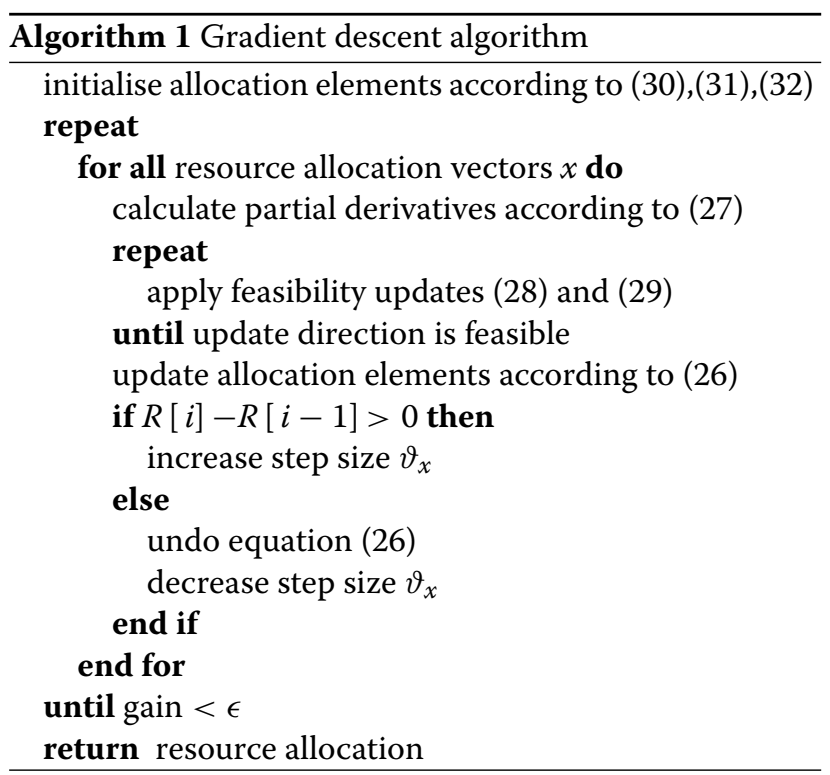


The result found by the gradient will therefore satisfy the integer constraint (24) even if time sharing allows $\mathrm{z}_{n, k}$ to be real valued. For high SNRs, however, $\mathrm{z}_{n, k}$ will not converge to integer values, but to a uniform distribution. In that case, we will choose those subcarriers that contribute the highest rates.

The modified gradient can be applied to the relaxed problems for the AF and CF protocols, even if the corresponding objectives are non-concave. It must be noted, however, that the solution might differ from the global optimum.

The obtained rates will be evaluated later in Section 6 .

\subsubsection{Subgradient method}

Due to the minimum and maximum terms in the rate functions (8) and (10), the gradient is not directly applied to the DF problems. The rate functions are not differentiable at all points, but the subgradients [37] can be defined nonetheless. As described in [38], a subgradient has to be applied here that adjusts the step size based on the iteration counter $i$. For implementation, a nonsummable diminishing step size [38] has been chosen:

$$
\vartheta_{x}=\frac{0.1}{\sqrt{i}} .
$$

Contrary to the above gradient, the updates according to (26) will not be undone if the objective becomes worse after an update. Thus, the implemented algorithm has to keep track of the best achieved sum rate and corresponding resource allocations:

$$
R_{\text {best }}^{\mathrm{DF}}[i]=\max \left\{R_{\text {best }}^{\mathrm{DF}}[i-1], R^{\mathrm{DF}}[i]\right\} .
$$

A common approach to improve the pace of convergence is to combine a bundle of previous subgradients to approximate the steepest descent direction [38]. The modified initialisation of the update variables (27) is then given by

$$
R_{\partial x}[i]=\alpha \frac{\partial R[i]}{\partial x}+(1-\alpha) R_{\partial x}[i-1],
$$

where $\alpha=\frac{1}{4}$ has been chosen for implementation. As the outlined algorithm will not adjust the step size to the slope of the objective, the convergence speed will be reduced dramatically.

\section{Carrier allocation}

Solving the global resource allocation problem is difficult due to the unknown subcarrier allocation sets $\mathbb{N}_{k}$. However, if optimal $\mathbb{N}_{k}$ would be known a priori, only the power allocations $\boldsymbol{p}^{\left(\mathcal{S}_{k}\right)}$ and $\boldsymbol{p}^{(\mathcal{R})}$ at the source and the relay node are left unknown. Thus, we will split the primal resource allocation problem into a separate CA problem and an individual PA problems.
This section deals with the optimisation of the CA assuming that the PAs are fixed:

$$
\left\{\mathbb{N}_{k}^{*}\right\}_{k=0}^{K-1}=\underset{\left\{\mathbb{N}_{k}\right\}_{k=0}^{K-1}}{\operatorname{argmax}} R .
$$

\subsection{Random carrier allocation}

The first approach assigns each subcarrier randomly to a specific user. If the probabilities are equal for all users, no CSI is required and the computational effort is low. On the other hand, the result might be far from the optimum. This approach serves as a lower bound.

\subsection{Improved greedy allocation}

As the name suggests, the CA immediately selects the best users for each subcarrier in a single step. The proposed approach consists of two steps, and the general idea is similar to [12]. All subcarriers are considered separately, and for each subcarrier $n$, the user $k$ with the best rate $r_{n, k}$ is chosen. To calculate specific rates $r_{n, k}$, the power is assumed to be uniformly distributed onto the subcarriers:

$$
\begin{aligned}
p_{n}^{\left(\mathcal{S}_{k}\right)}=\frac{1}{N / K} P_{\max } & \forall n \in \mathbb{N}, k \in \mathbb{K} \\
p_{n}^{(\mathcal{R})}=\frac{1}{N} P_{\max } & \forall n \in \mathbb{N} .
\end{aligned}
$$

This PA is of course not the optimal one. Actually, it might not even be feasible, but it nevertheless ensures a fair comparison of users.

The algorithm described so far will often fail to find the optimal CA. If the channel coefficients are similar for different users, the selection of an inferior subcarrier instead of the best subcarrier might be beneficial. To clarify this observation, we would like to give an extreme example. In the case of an empty set $\mathbb{N}_{k}=\emptyset$, the source node $\mathcal{S}_{k}$ will not get any subcarrier. However, if no subcarrier is assigned to $\mathcal{S}_{k}$, its available transmit power cannot be used to improve the sum rate $R$.

Therefore, some kind of post-processing is required that checks for possible improvements of sum rate $R$. After the initial (greedy) assignment, the improved Greedy CA searches for subcarriers that should be reassigned to other source nodes. A subcarrier will be moved to a different allocation set $\mathbb{N}_{k}$ if this leads to an improvement in the sum rate $R$.

To keep computational effort low, the available power will be distributed uniformly among subcarriers for the computation of rates $r_{n, k}$. The proposed algorithm is shown as a pseudo-code in Algorithm 2. The greedy part and each iteration of the second part require $N K$ steps for computation, but the second part is repeated until no further improvement can be achieved. If it is assumed that each subcarrier is assigned to each user at most once in the second part, the computational effort of the improved Greedy CA is limited to $\mathcal{O}\left(N^{2} K^{2}\right)$. 


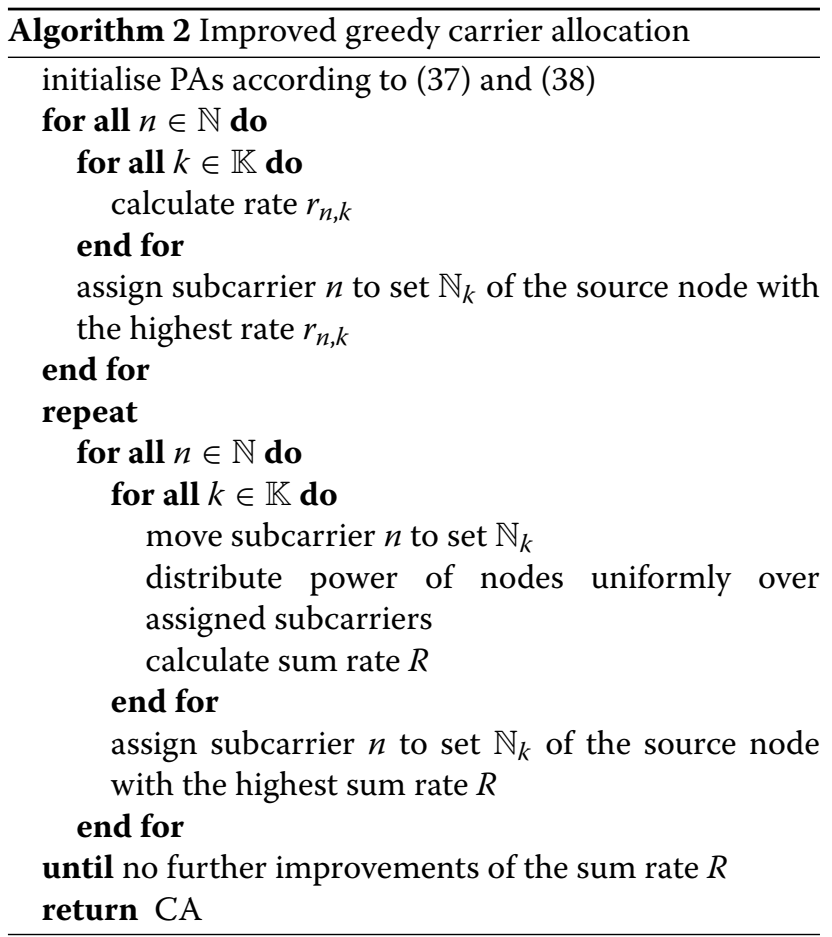

\subsection{Hungarian carrier allocation}

The next approach is based on the Hungarian algorithm [39] that solves arbitrary quadratic assignment problems in polynomial time.

If there are, for example, $n$ persons and $n$ jobs to be done, the quadratic matrix

$$
\left[\begin{array}{cccc}
m_{0,0} & m_{0,1} & \cdots & m_{0, n} \\
m_{1,0} & m_{1,1} & \cdots & m_{1, n} \\
\vdots & \vdots & \ddots & \vdots \\
m_{n, 0} & m_{n, 1} & \cdots & m_{n, n}
\end{array}\right]
$$

describes the cost matrix, where element $m_{i, j}$ corresponds to the cost of person $i$ doing job $j$. If each person is supposed to do exactly one job, the allocation problem is to find the exclusive assignment matrix $\mathbf{Z}$ that minimises the sum over the costs of all jobs:

$$
\begin{array}{rlrl} 
& \mathbf{Z}^{*}=\underset{\mathbf{Z}}{\operatorname{argmin}} \sum_{i=0}^{n-1} \sum_{j=0}^{n-1} \mathrm{z}_{i, j} m_{i, j} \\
\text { s.t. } \quad & \sum_{i=0}^{n-1} \mathrm{z}_{i, j}=1 & \forall 0 \leq j \leq n-1 \\
& \sum_{j=0}^{n-1} \mathrm{z}_{i, j}=1 & \forall 0 \leq i \leq n-1 \\
\mathrm{z}_{i, j} \in\{0,1\} & \forall 0 \leq i \leq n-1, \\
& & 0 \leq j \leq n-1 .
\end{array}
$$

The trivial solution of this problem is to check all $n$ ! possible assignments and choose the set with the lowest total costs. The Hungarian algorithm proposed in [39] solves this problem in $\mathcal{O}\left(n^{4}\right)$ time. Further modifications of the algorithm lead to a reduction to $\mathcal{O}\left(n^{3}\right)$ [40].

If it is possible to apply the algorithm to the integer problem within this work, it might be possible to reduce the exponential effort required to do the exhaustive. The Hungarian algorithm can be applied to our problem if the persons are replaced by source nodes $\mathcal{S}_{k}$ and the jobs are replaced by the $N$ subcarriers. The cost elements correspond to the negative rate $-r_{n, k}$ that a source node $\mathcal{S}_{k}$ can achieve on subcarrier $n$ :

$$
m_{n, k}=-r_{n, k}
$$

However, the knowledge of optimal PA vectors $p_{n}^{\left(\mathcal{S}_{k}\right)}$ and $p_{n}^{(\mathcal{R})}$ is required to compute the costs $-r_{n, k}$. Similar to the greedy approach above, the PAs are assumed to equal the average values (37) and (38). The Hungarian algorithm in itself is restricted to the solution of quadratic assignment problems, whereas the cost matrix of the CA problem is of size $N \times K$. If there are more subcarriers than users $N>K$, multiple subcarriers can be assigned to the same user.

However, if the number of subcarriers per source node $\left|\mathbb{N}_{k}\right|$ is known, a valid cost matrix can be found

$$
\left[\begin{array}{ccccc}
\cdots & -r_{0, k} & \cdots & -r_{0, k} & \cdots \\
\cdots & -r_{1, k} & \cdots & -r_{1, k} & \cdots \\
\cdots & \vdots & & \vdots & \\
\cdots & \underbrace{-r_{N-1, k}}_{\left|\mathbb{N}_{k}\right|} \cdots & -r_{N-1, k} & \cdots
\end{array}\right],
$$

where the rates $r_{n, k}$ of each source node $\mathcal{S}_{k}$ might be listed multiple times in $\left|\mathbb{N}_{k}\right|$ columns. That way the cost matrix is stretched to size $N \times N$.

As the number of subcarriers per user is not a priori known, the Hungarian algorithm has to solve several problems, one for each combination $\left\{\left|\mathbb{N}_{0}\right|, \cdots,\left|\mathbb{N}_{K-1}\right|\right\}$. This will be clarified based on an example. For $K=2$ source nodes and $N=4$ subcarriers, there are five possible combinations of $\left\{\left|\mathbb{N}_{0}\right|,\left|\mathbb{N}_{1}\right|\right\}:\{4,0\},\{3,1\},\{2,2\},\{1,3\}$ and $\{0,4\}$. For the first one, the Hungarian algorithm will assign all subcarriers to source node $\mathcal{S}_{0}$. In the second case, only three subcarriers will be assigned to $\mathcal{S}_{0}$, but one subcarrier will be assigned to $\mathcal{S}_{1}$. Finally, the sum rates must be calculated for all of these five possibilities, and the best one is chosen as the solution.

At first glance, this seems to be similar to the primal exhaustive search problem, but as the Hungarian algorithm finds the best allocation, the ordering of columns is irrelevant in the cost matrices. In the considered example, there are five possible cost matrices that have to be optimised by the Hungarian algorithm. Contrary, an 
exhaustive search has to check $K^{N}=2^{4}=16$ possible subcarrier allocations.

In general, the number of possible cost matrices is given by $\frac{(N+K-1) !}{(K-1) !(N) !}$. Since the Hungarian algorithm itself is bounded above by $\mathcal{O}\left(N^{3}\right)$, the maximum overall effort is below $\mathcal{O}\left(N^{K}\right)$. For $N \gg K$, this is much lower than the effort $\mathcal{O}\left(K^{N}\right)$ required for an exhaustive search. However, the complexity is still much higher than the effort of the greedy approach.

The entire algorithm is summarised as a pseudo-code in Algorithm 3.

\section{Power allocation}

The general resource allocation problem has been defined in (14) to (20). However, if sets $\mathbb{N}_{k}$ have been obtained by one of the proposed CA algorithms, only the power allocation vectors are left unknown.

\subsection{Equal power allocation}

The first approach is straightforward and does not require any CSI. For each node, the available power $P_{\max }$ is distributed uniformly over allocated subcarriers:

$$
\begin{array}{ll}
p_{n}^{\left(\mathcal{S}_{k}\right)}=\frac{P_{\max }}{\left|\mathbb{N}_{k}\right|} & \forall k \in \mathbb{K}, n \in \mathbb{N}_{k} \\
p_{n}^{(\mathcal{R})}=\frac{P_{\max }}{|\mathbb{N}|} & \forall n \in \mathbb{N} .
\end{array}
$$

\subsection{Separate power allocation}

For the direct point-to-point transmission over $n$ orthogonal channels, the PA is done optimally by the well-known water-filling solution. It can be derived by dual optimisation, since the duality gap becomes zero for convex optimisation problems. However, this solution is not valid for the relay network. If there is a relay node, the PA

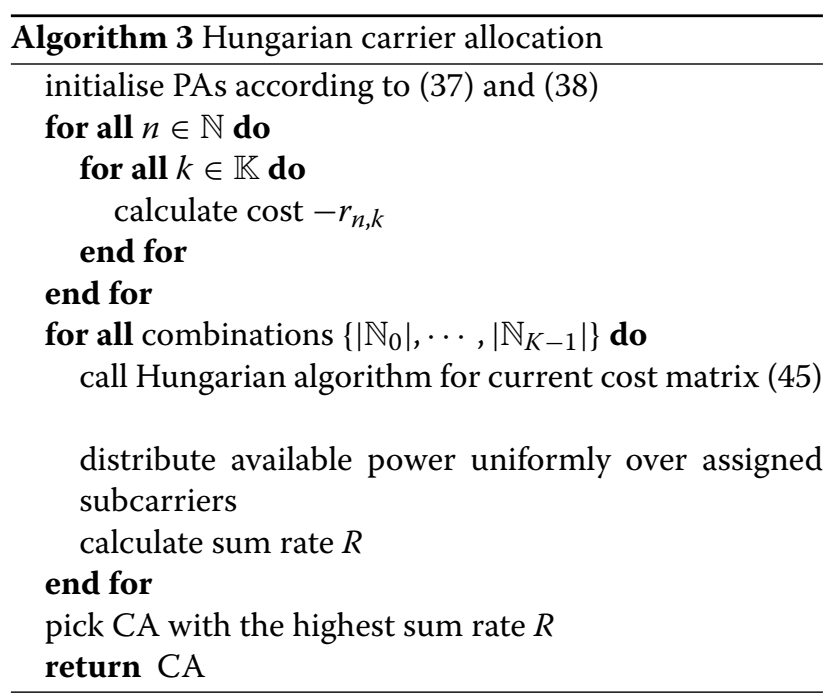

depends on the protocol used at that relay node and the PA problem is not necessarily convex.

Another issue is the number of power constraints. As this work is restricted to the case of individual power constraints, each additional user will add a new constraint to the optimisation problem. The number of Lagrange multipliers in the dual optimisation therefore depends on the number of source nodes $K$. A general solution of such a dual optimisation problem for $K$ users is therefore hard to find. Even if we assume that each additional Lagrange multiplier can be found with an additional search over that parameter, the computational effort will increase exponentially with the number of source nodes $K$. Furthermore, the duality gap would not necessarily be zero if the problem is not concave.

To solve the PA problem for all nodes, we will split the problem into $K+1$ subproblems. There is one individual subproblem for each power constraint:

$$
\begin{aligned}
\boldsymbol{p}^{*(\mathcal{R})}=\underset{\boldsymbol{p}^{(\mathcal{R})}}{\operatorname{argmax}} & R \\
\boldsymbol{p}^{*\left(\mathcal{S}_{k}\right)}=\underset{\boldsymbol{p}^{\left(\mathcal{S}_{k}\right)}}{\operatorname{argmax}} & R
\end{aligned} \quad \forall k \in \mathbb{K} .
$$

If the power allocations of the other nodes are fixed, the remaining subproblem will be concave for all of the considered relay protocols. They can therefore be solved optimally by dual optimisation. A detailed derivation of the solutions $\boldsymbol{p}^{*(\mathcal{R})}$ and $\boldsymbol{p}^{*\left(\mathcal{S}_{k}\right)}$ for the considered relay protocols can be found in the Appendix.

A global power allocation solution can be obtained by solving the subproblem of all nodes iteratively. Then, a solution $\boldsymbol{p}^{\left(\mathcal{S}_{k}\right)}$ for node $\mathcal{S}_{k}$ can be used as a constant value in the next subproblem of node $\mathcal{S}_{k+1}$. Hammerström et al. suggested in [22] that the uniform power distribution is a good initial starting point for an iterative algorithm. This iterative process can be repeated until the result is good enough or no further significant gain can be obtained. The proposed algorithm is summarised as a pseudo-code in Algorithm 4.

Next, we will have a look at the convergence behaviour of this approach. In general, the PA is only guaranteed to converge to an unambiguous PA if the global PA problem

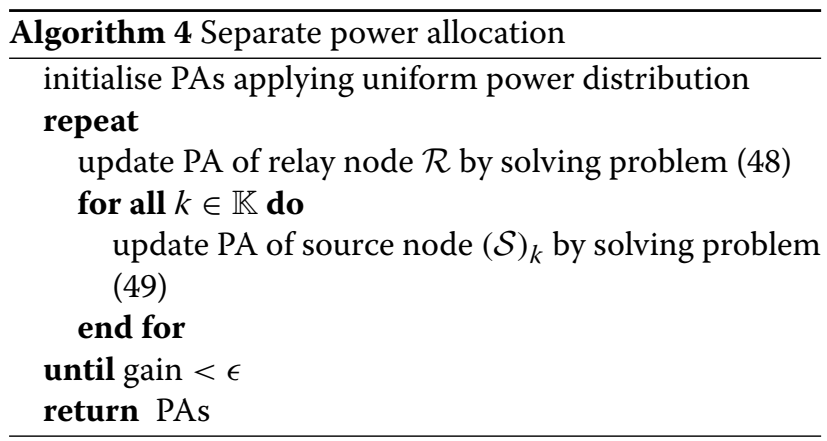


is concave. However, if the global PA would be concave, dual optimisation can be applied directly. Nevertheless, although the global PA problem is not concave, simulations indicate that solving subproblems (48) and (49) iteratively leads to convergence after few iterations. Figure 2 shows the the convergence of Algorithm 4. The number of optimisation cycles refers to the number of iterations in the REPEAT-UNTIL loop in Algorithm 4, and the relative sum rates are normalised to the obtained sum rate after convergence has been reached.

\subsubsection{Decode and forward}

For the DF protocols, the sum rates obtained by solving subproblems iteratively are significantly worse than the results obtained by a gradient approach. As described earlier, this is due to the hard borders caused by the minimum and maximum terms in the rate functions. We will thus extend the algorithm for the DF protocols. In accordance with the bundle approach of the subgradient method, the process of convergence is slowed by applying a simple filter with infinite impulse response to the results $p_{n}^{*(\mathcal{A})}[i]$ of the separate power optimisations:

$$
\begin{aligned}
p_{n}^{(\mathcal{A})}[i]= & \alpha p_{n}^{*(\mathcal{A})}[i]+(\alpha-1) p_{n}^{(\mathcal{A})}[i-1] \\
& \forall \mathcal{A} \in\left\{\mathcal{R}, \mathcal{S}_{0}, \ldots, \mathcal{S}_{K-1}\right\} .
\end{aligned}
$$

\section{Results}

In this section, the proposed algorithms will be analysed and compared w.r.t the achieved sum rates and the computational effort. For the compound approaches, CA is done first and only once. The PA is also performed only once using the previously obtained CA.

We will use a basic set-up but vary different simulation parameters such as the position of nodes, the SNR and the number of subcarriers $N$. Unless otherwise specified, there is a MARC with $K=2$ source nodes. The source and destination nodes form a normalised, equilateral triangle as depicted in Figure 3. For the analysis, a constant path

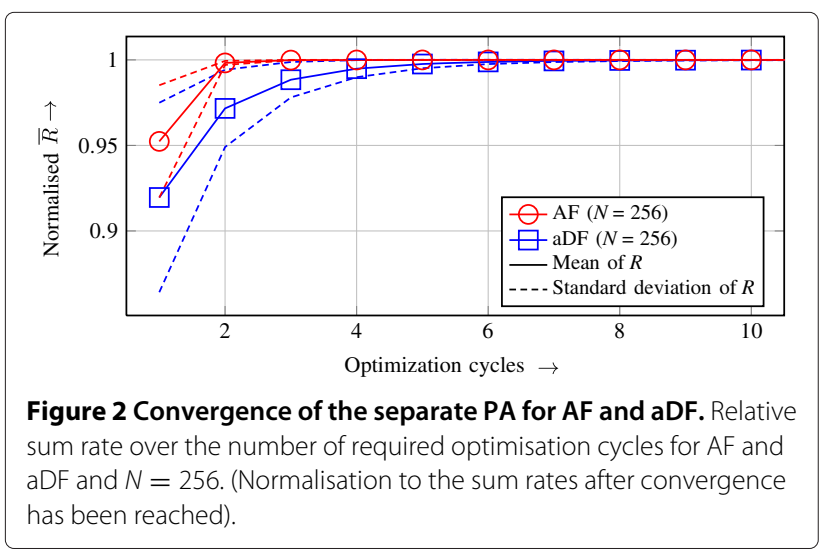

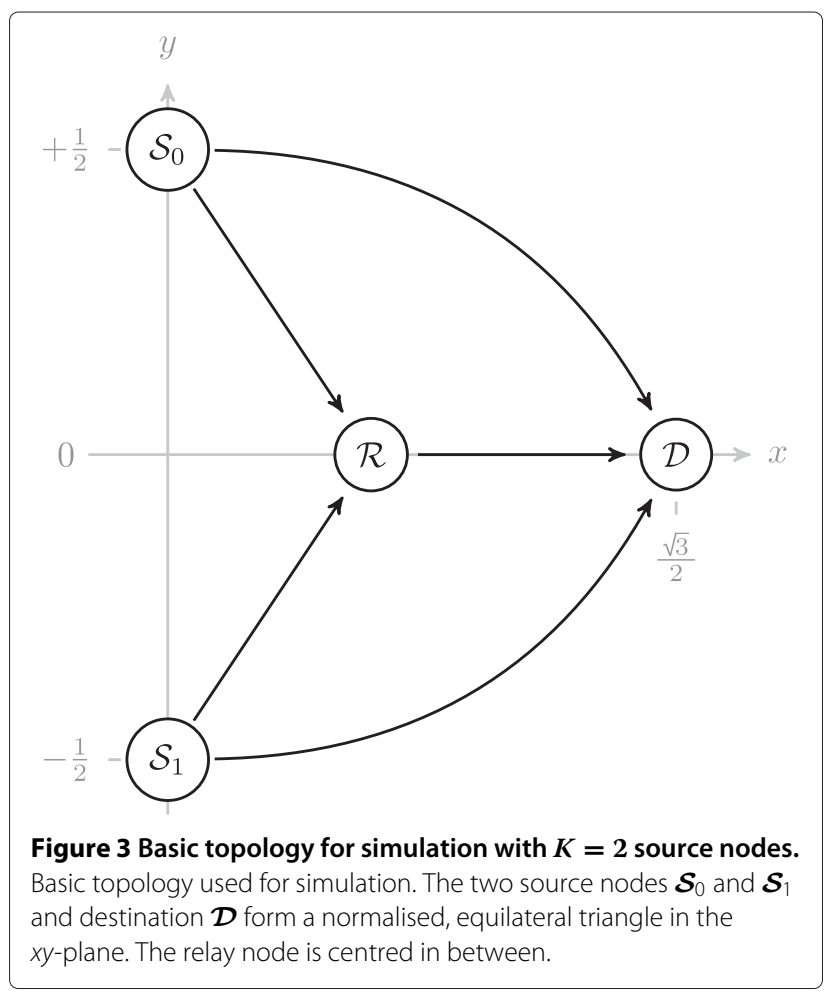

loss exponent $\alpha=4$ is assumed that is valid for urban areas with high attenuations [41, Chapter 3$]$. The number of subcarriers is $N=4$, and $P_{\max }$ is chosen such that the average SNR between $\mathcal{S}_{0}$ and $\mathcal{D}$ becomes $E\left\{\gamma_{n}^{\left(\mathcal{S}_{0} \mathcal{D}\right)}\right\}=1 \widehat{=}$ $0 d B$. This average SNR for the direct path has been chosen low, such that the application of a relay is reasonable.

Most of the presented numerical results will be restricted to the $\mathrm{AF}$ and $\mathrm{nDF}$ protocols as results will not differ much for $\mathrm{CF}$ and aDF, respectively. Of course, the achieved sum rates are not identical due to the different objectives in the optimisation problems, but the relations between the curves of the proposed algorithms are similar and conclusions are thus almost identical.

\subsection{Relay position}

First, we compare the obtained sum rates for different relay positions, i.e. we move the relay node $\mathcal{R}$ in horizontal direction. Figure 4 shows the average sum rates over the normalised relay position $x_{\mathcal{R}} / x_{\mathcal{D}}$ for AF. In general, the best results can be achieved for relay positions in the centre between source and destination nodes. For this relay position, the maximum length of the indirect paths is minimised. As the AF strategy amplifies the noise received at the relay, a medium SNR for both paths $\mathcal{S} \rightarrow \mathcal{R}$ and $\mathcal{R} \rightarrow \mathcal{D}$ achieves much better results than the combination of a good and a bad SNR. As the problem size $N=4$ is sufficiently small, it is possible to do an exhaustive search 


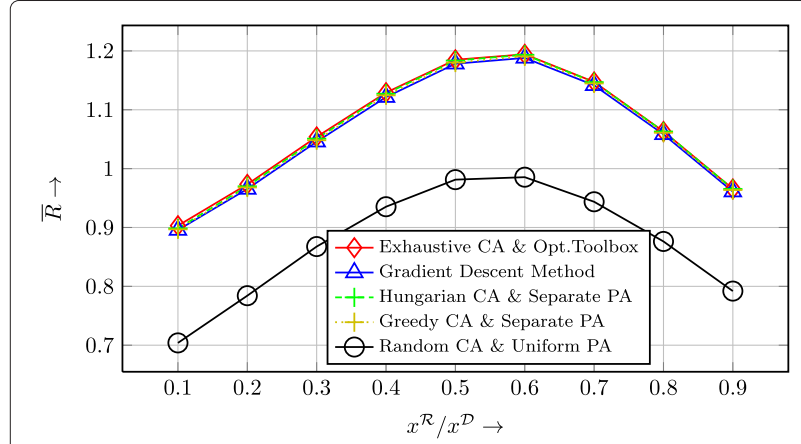

Figure 4 Average sum rates over normalised relay position for $\mathrm{AF}$ and $N=4$.

over all $2^{4}$ possible subcarrier allocations. If the Optimization Toolbox is used to determine the PA for each possible CA, the upper curve in Figure 4 will be achieved. All the other algorithms perform worse. However, the gap to the results of CA and PA, Hungarian CA and PA or the gradient is at most $0.5 \%$ and, thus, very small. Contrary, the combination of random CA and uniform PA performs significantly worse. This is also as expected, since no CSI is used in that approach.

In Figure 5, the achievable AF sum rates are depicted for a bigger problem with $N=256$ subcarriers. The results of the exhaustive and Optimization Toolbox are not presented since those algorithms cannot find a solution in reasonable time. Compared to the results for $N=4$, the achievable sum rates are slightly higher due to the increased frequency diversity. However, the algorithm that does not use any CSI cannot profit from diversity.

Another important observation is that the influence of the CA seems to be much higher than the influence of the PA. To illustrate this, two additional curves have been plotted in Figure 5. The random CA and PA show the sum rate for the case that only the transmit power is optimised.

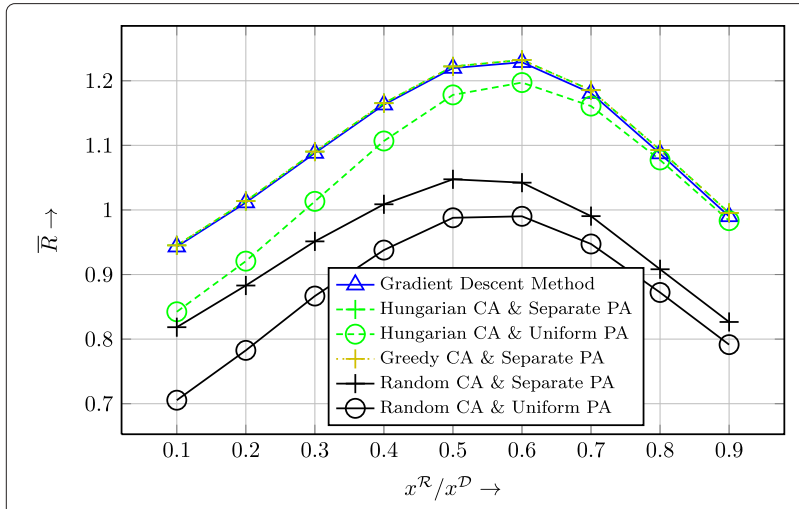

Figure 5 Average sum rates over normalised relay position for AF and $N=256$.
The Hungarian CA and uniform PA depict the impact of the CA if a uniform power distribution is applied. For the given scenario, the gain of the $\mathrm{CA}$ is considerably higher than the gain achieved by the PA. The reason is that the CA selects channels that are well suited for transmission. If a channel coefficient is bad for a specific user, it is quite probable that the subcarrier will be assigned to a another user with a better channel coefficient. Hence, CA removes worst channels from the optimisation problem. The PA, on the other hand, has to deal with assigned subcarriers, no matter how good or bad. If there is a subcarrier with a very bad channel coefficient, all PA can do is assigning only few transmit power or ignoring that channel. Figure 6 illustrates that similar results are obtained for $\mathrm{nDF}$. The main difference is that DF can outperform AF if the relay is positioned close to the source node, but AF is the better choice if the relay is close to the destination.

For a comparison of all of the considered relay protocols, the obtained rates of Hungarian CA and PA are plotted in Figure 7 for $\mathrm{AF}, \mathrm{nDF}, \mathrm{aDF}$ and $\mathrm{CF}$ strategies. The figure shows that the gain of the adaptive DF protocol over the non-adaptive DF strategy is quite small. The reason is that turning off the relay in aDF is only necessary due to fading. If the relay is positioned between the source nodes and destination, the restriction of $\mathrm{nDF}$ to the $\mathcal{S} \rightarrow \mathcal{R}$ path is not significant as the $\mathcal{S} \rightarrow \mathcal{R}$ path is much shorter than the direct $\mathcal{S} \rightarrow \mathcal{D}$ path.

If the relay moves close to the destination node $x^{(\mathcal{R})} / x^{(\mathcal{D})}>0.6$, the results for DF drop below the sum rates obtained for AF and CF. These approaches do not suffer that much from a long $\mathcal{S} \rightarrow \mathcal{R}$ path, since successful decoding at the relay is not required. On the other hand, both approaches have to deal with the additional noise obtained at the relay. It is not removed at the relay as in the DF strategy, but it is forwarded. The gain of CF compared to that of AF can be explained by the fact that CF does not amplify the noise obtained at the relay node.

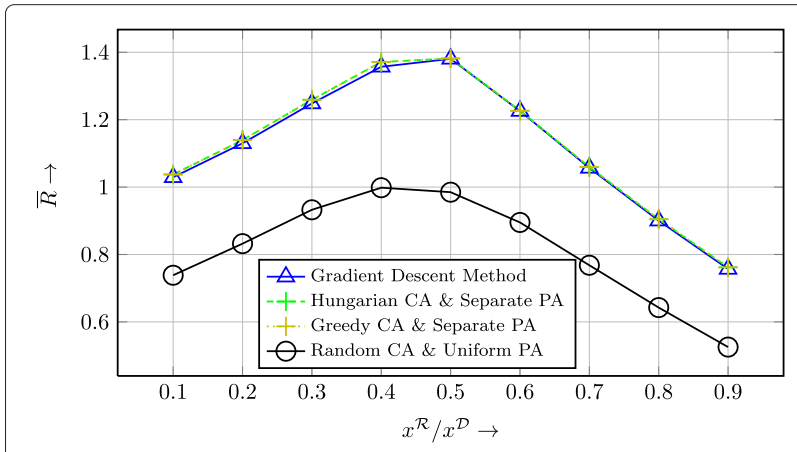

Figure 6 Average sum rates over normalised relay position for nDF and $N=256$. 


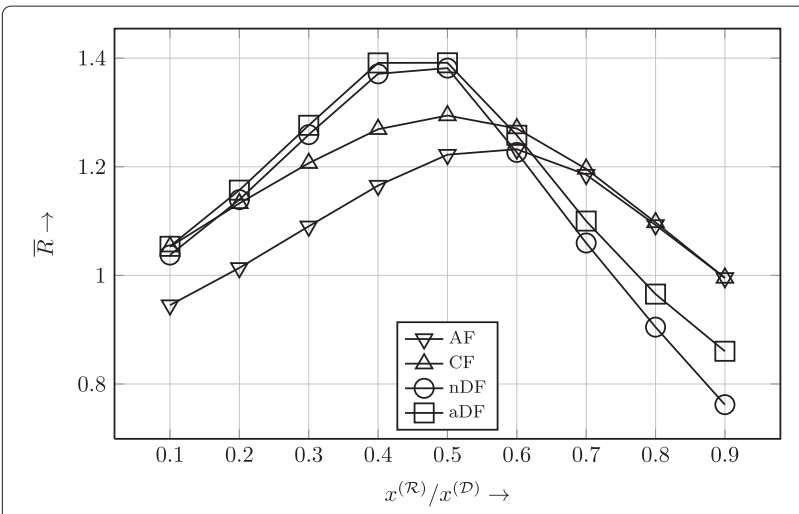

Figure 7 Comparison of the average sum rates for $N=256$ and different relay protocols.

\subsection{Source position}

Next, we will vary the position of source node $\mathcal{S}_{1}$ along the direction towards the destination $\mathcal{D}$. Note that distance $d_{\left(\mathcal{S}_{1} \mathcal{D}\right)}$ changes, but the position of source node $\mathcal{S}_{0}$ and thus $d_{\left(\mathcal{S}_{0} \mathcal{D}\right)}$ is still fixed. Figure 8 shows for AF that the algorithms perform similarly well, even if the path distances differ for the source nodes. If source node $\mathcal{S}_{1}$ moves close to the destination $\left(d_{\left(\mathcal{S}_{1} \mathcal{D}\right)} \ll 1\right)$, sum rates increase rapidly. However, the distribution of transmission rates on the source nodes becomes unfair in that case. The reason is that the SNR of the direct path $\mathcal{S}_{1} \rightarrow \mathcal{D}$ becomes also very large and the maximum sum rate can be achieved if all subcarriers are assigned to source node $\mathcal{S}_{1}$. However, long-term fairness might still be achieved among the source nodes if priority weights are added for each user [42].

\subsection{Signal-to-noise ratio}

Next, we will use a fixed position for all nodes but vary the average SNRs. According to (4), the power constraint $P_{\max }$ will be chosen such that the average SNR of the direct path becomes $E\left\{\gamma_{n}^{\left(\mathcal{S}_{k} \mathcal{D}\right)}\right\}$. For nDF, Figure 9 shows similar

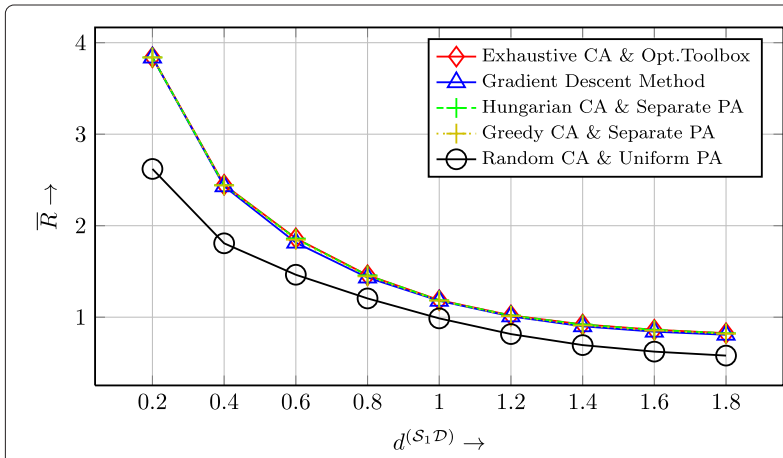

Figure 8 Average sum rates over different positions of second source node $\mathcal{S}_{1}$ for $\mathrm{AF}$ and $N=4$.

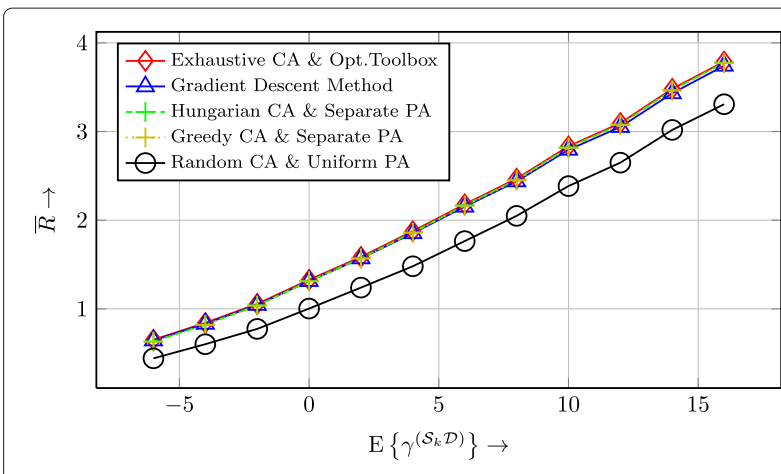

Figure 9 Average sum rates over different SNR values, $\mathrm{nDF}$ and $N=4$.

results as the variation of the source node $\mathcal{S}_{1}$. The gaps between the obtained results stay almost constant, but the achievable sum rate increases significantly for increasing SNR. This is equivalent to the observation that the relative gain to a uniform resource allocation decreases. If the SNR is large, the resource allocation has only a minor influence on achievable rates. On the other hand, if the SNR is low, the proposed resource allocations can improve achievable transmission rates significantly.

\subsection{Number of subcarriers}

Figure 10 depicts the achievable sum rate $R$ of the AF strategy over the number of subcarriers $N$. Since the exhaustive is only feasible for small problems, only results for small $N$ are shown. On the other hand, Figure 10 indicates that the obtained solutions are close to the optimal results of the exhaustive.

Even if the achieved sum rates are almost identical for the proposed algorithms and large $N$, the approaches are not equally well suited to solve the resource allocation problem. Figure 11 shows the required number of iterations of the different algorithms for AF and different problem sizes $N$. Since the number of iterations as well as the number of subcarriers $N$ is scaled logarithmically, the slope of the curves corresponds to the average computational effort of the algorithms and a vertical offset corresponds to a constant factor. This illustration does not

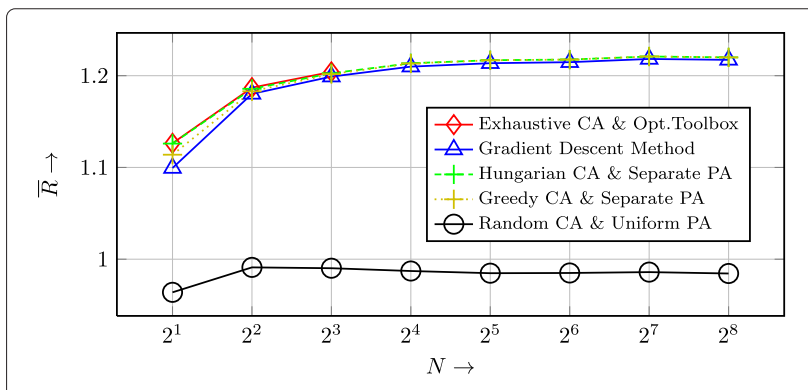

Figure 10 Average sum rates of AF over different $N$. 


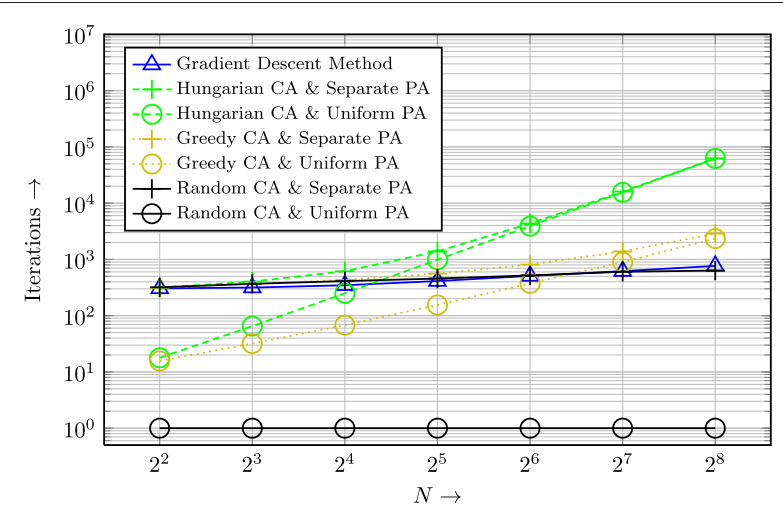

Figure 11 Average number of required iterations over the number of subcarriers $N$ for AF.

depict the real computational effort of the algorithms as the effort per single iteration is neglected and the slopes of the obtained curves are thus too small. However, if the computational effort of a single iteration is assumed to be $\mathcal{O}(N K)$, the slopes of the curves are a good approximation of the average effort.

Compared to the other approaches, the required effort of the random CA and uniform PA is the best as the assignment of PAs can be done in a single iteration. On the other hand, the corresponding sum rates are by far the worst. The other algorithms require significantly more iterations. The effort of the compound approaches that do the CA first (Hungarian CA or improved Greedy CA) and a PA afterwards can be interpreted as combination of CA and PA efforts. The slope of the Hungarian CA and uniform PA is, for example, much steeper than the increase of the random CA and PA. The combination of both approaches (Hungarian CA and PA) is thus limited by the slope of the Hungarian CA. (The curve converges to the effort of Hungarian CA and uniform PA for large N.) As the increase in the logarithmic plot is linear, these algorithms are able to solve the combinatorial optimisation problem in polynomial time.

The improved Greedy CA leads to a similar estimate of the effort, but the increase is much slower. The improved Greedy CA has therefore a lower average effort, even if achieved sum rates are almost identical. Finally, Figure 11 also shows the result of the straightforward gradient. The slope corresponding to the gradient method is even smaller than the slope of the greedy approach. For AF, the gradient achieves therefore the best performance among the proposed algorithms. Its number of iterations increases not even linearly with the number of subcarriers $N$, while the obtained results are at least as good as the results of the other proposed schemes. For the DF case, however, this is not true. Due to the subgradient, the average effort is much worse.

\subsection{Number of source nodes}

Finally, the number of source nodes will be increased. For the simulation of an example topology, it is assumed that $K=4$ source nodes are positioned regularly in a cluster at $x=0$. The modified topology is depicted in Figure 12 .

The results depicted in Figure 13 for the AF protocol are similar to the results obtained for $K=2$ nodes in Figure 4, but the achievable average rates are slightly higher due to the increased number of the additional source nodes and possible paths. Furthermore, the optimal position of the relay is slightly shifted to the destination node, and there is a bigger gap between the suboptimal approaches and the exhaustive search.

Figure 14 shows results for the same set-up with $K=4$ sources but $N=16$ subcarriers. Even if there is only a slight increase in the number of subcarriers, an exhaustive cannot be done in reasonable time for this scenario. Figure 14 nonetheless shows that the losses of the suboptimal approaches (again) decrease if the number of subcarriers increases.

\section{Conclusions}

Within this work, several algorithms for resource allocation in a relay-assisted OFDMA system have been proposed. Here, sub-CA and PA are optimised separately or jointly (JA) to maximise the sum rate over all subcarriers. These approaches have been applied to several relay protocols, such as $\mathrm{AF}$, adaptive and non-adaptive $\mathrm{DF}$ and $\mathrm{CF}$, and the achieved sum rates, convergence and

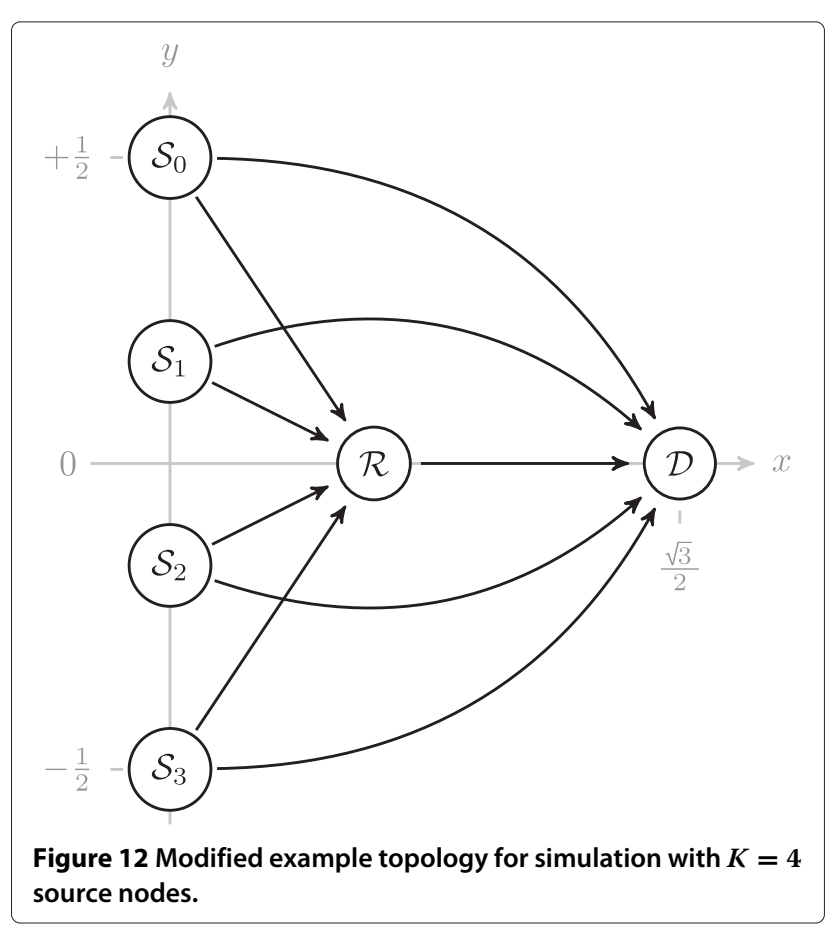




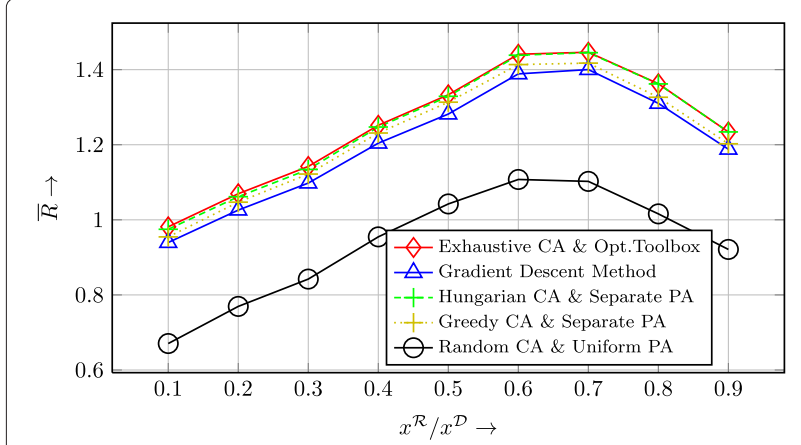

Figure 13 Average sum rates over normalised relay position for $\mathrm{AF}, N=4$ and $K=4$ source nodes.

computational effort have been evaluated by numerical simulation.

Finally, we will summarise the obtained results for each of the proposed algorithms.

\subsection{Random CA and uniform PA}

The first straightforward approach does not use any CSI to optimise resource allocation, but it randomly assigns subcarriers and distributes the transmit power uniformly. On the one hand, this algorithm has the lowest computational effort. On the other hand, no optimisation is done, and the achieved sum rates are a lower bound. Certainly, it is possible to do worse, but this is the best strategy without using CSI. All other algorithms that use at least some CSI can outperform this approach in terms of sum rates. However, if the SNR is large, the loss becomes small.

\subsection{Exhaustive search and Optimization Toolbox}

The second approach is quite the opposite of the first approach. The combinatorial CA problem is tackled by an exhaustive that checks all possible subcarrier assignments.

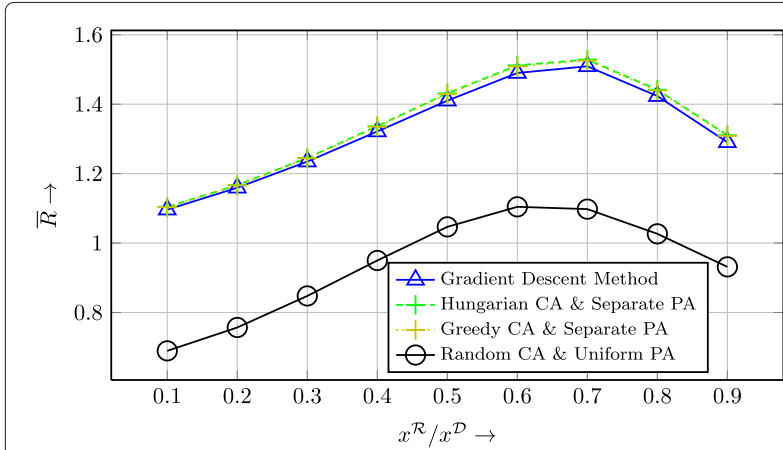

Figure 14 Average sum rates over normalised relay position for $\mathrm{AF}, N=16$ and $K=4$ source nodes.
The PA is determined by several calls of Optimization Toolbox in Matlab with varying initial values.

This approach cannot claim to find the global optimal solution, but it is assumed to be very close to the optimum. The disadvantage of this algorithm is of course its huge computational effort. For $K=2$ and $N=4$, an exhaustive might be reasonable, but for a realistic problem size, this approach is infeasible.

\subsection{Hungarian CA and separate PA}

This approach is able to reduce the number of tests in the exhaustive by applying the Hungarian algorithm to the CA problem. However, this algorithm is suboptimal since Hungarian CA assumes uniformly distributed transmit power for all subcarriers. Nevertheless, it finds almost always the same CAs as the exhaustive for all considered relay protocols, but it requires considerable less effort. Compared to that of the CA, the computational effort of the Hungarian $\mathrm{CA}$ is still high. If the number of users is limited to two source nodes, the computational effort might be reasonable, even for practical numbers of subcarriers. For larger networks with many source nodes, the computational effort is also known to be infeasible.

The PA is performed after a CA has been found. The PA problem is split into individual, but concave, subproblems, and these separate subproblems can be solved optimally. The PA solutions have been determined for the considered relay strategy individually, by solving the corresponding dual optimisation problems. Numerical results show that the iterative optimisation converges to a solution within few iterations. The average computational effort of the PA is low for all relay strategies, and it increases only slowly for larger problems.

\subsection{Improved greedy CA and separate PA}

This approach achieves almost the same sum rates as the previous approach, but it leads to a lower computational effort. Instead of the expensive search performed by Hungarian CA, this algorithm uses a greedy approach.

The approach achieves the best trade-off between achieved sum rates and computational effort.

\subsection{Gradient descent method}

The last approach is an attempt to solve the resource optimisation problem with a straightforward gradient. The exclusive CAs are relaxed, but the proposed algorithm converges to the exclusive CAs within few iterations if the SNR is not too high. This algorithm is therefore even suitable if time sharing is not reasonable.

It is obvious that the results found by this algorithm are not necessarily optimal if the problem is not concave. However, numerical simulation indicates that this algorithm will converge to very good results if random CA and 
uniform PA are used as initial values. Furthermore, the relation between achieved sum rates and computational effort is by far the best for the AF and the CF case. Here, only random CA and uniform PA achieve a better effort.

For DF strategies, however, the gradient leads to some convergence issues. Due to the minimum term within the rate functions, the gradient has to be replaced by a subgradient that is known to converge much slower.

\section{Appendix}

In the following paragraphs, the solutions of the PA subproblems will be derived for all of the considered relay protocols.

However, separate power optimisation leads to an unresolved issue concerning the notation. So far, power allocation vectors $\boldsymbol{p}^{\left(\mathcal{S}_{k}\right)}$ refer to the individual power allocation of a single node. Therefore, the elements $p_{n}^{\left(\mathcal{S}_{k}\right)}$ correspond only to source node $\mathcal{S}_{k}$ and are zero for $n \notin \mathbb{N}_{k}$. Contrary, each element in $\boldsymbol{p}^{(\mathcal{R})}$ may refer to a different source node $\mathcal{S}_{k}$ as subcarriers at the relay node can be used by different users.

For the sake of clarity, an alternative representation will be introduced here. If CAs are exclusive and fixed, each subcarrier $n$ corresponds to exactly one source node $\mathcal{S}_{k}$ with $n \in \mathbb{N}_{k}$. The index $k$ is therefore redundant and can be omitted in several notations:

$$
\begin{array}{rlrl}
p_{n}^{\left(\mathcal{S}_{k}\right)} & \rightarrow \check{p}_{n}^{(\mathcal{S})} & & \forall n \in \mathbb{N}_{k} \\
\lambda_{n}^{\left(\mathcal{S}_{k} \mathcal{B}\right)} \rightarrow \check{\lambda}_{n}^{(\mathcal{S B})} & \forall n \in \mathbb{N}_{k} \\
r_{n, k} \rightarrow \check{r}_{n} & \forall n \in \mathbb{N}_{k} .
\end{array}
$$

\section{Amplify and forward}

The proposed solution for the AF protocol is an improvement of the one proposed by Hammerström and Wittneben in [22], where the authors consider the single user case with individual power constraints for the source and the relay node. Furthermore, they assume that the destination $\mathcal{D}$ is not able to receive any information directly. This shadowing is equivalent to a channel-tonoise ratio of $\lambda_{n}^{\left(\mathcal{S}_{k} \mathcal{D}\right)}=0$. Therefore, the scenario in [22] is a special case of the power allocation problem considered here. The AF solution without simplifications has already been proposed in [43], but it will be summarised here nonetheless for the sake of completeness.

Solving the separate optimisation problems (48) and (49) leads to a quadratic function with two solutions for the relay node and a cubic function with three solutions for each source node. However, since channel coefficients, as well as power vectors, are real valued and non-negative, only a single solution is valid in all cases.

The relay solution is given by

$$
\begin{aligned}
& A_{2}=\frac{\left(\check{\lambda}_{n}^{(\mathcal{S R})} \check{p}_{n}^{(\mathcal{S})}+1\right)\left(2 \check{p}_{n}^{(\mathcal{S D})} \check{p}_{n}^{(\mathcal{S})}+\check{\lambda}_{n}^{(\mathcal{S R})} \check{p}_{n}^{(\mathcal{S})}+2\right)}{2 \lambda_{n}^{(\mathcal{R D})}\left(\check{p}_{n}^{(\mathcal{S D})} \check{p}_{n}^{(\mathcal{S})}+\check{\lambda}_{n}^{(\mathcal{S R})} \check{p}_{n}^{(\mathcal{S})}+1\right)} \\
& p_{n}^{*(\mathcal{R})}=\left[A_{1}-A_{2}\right]^{+},
\end{aligned}
$$

where $[\cdot]^{+}$is equivalent to $\max \{0, \cdot\}$.

The solution for the source nodes is given by

$$
\begin{aligned}
& B_{1}=\check{\lambda}_{n}^{(\mathcal{S D})}-v^{*}+\check{\lambda}_{n}^{(\mathcal{S D})} \lambda_{n}^{(\mathcal{R} \mathcal{D})^{2}} p_{n}^{(\mathcal{R})^{2}} \\
& +\check{\lambda}_{n}^{(\mathcal{S R})} \lambda_{n}^{(\mathcal{R D})^{2}} p_{n}^{(\mathcal{R})^{2}}-\lambda_{n}^{(\mathcal{R} \mathcal{D})^{2}} v^{*} p_{n}^{(\mathcal{R})^{2}} \\
& +2 \check{\lambda}_{n}^{(\mathcal{S D})} \lambda_{n}^{(\mathcal{R D})} p_{n}^{(\mathcal{R})}+\check{\lambda}_{n}^{(\mathcal{S R})} \lambda_{n}^{(\mathcal{R D})} p_{n}^{(\mathcal{R})} \\
& -2 \lambda_{n}^{(\mathcal{R} D)} v^{*} p_{n}^{(\mathcal{R})} \\
& B_{2}=\check{\lambda}_{n}^{(S \mathcal{D})} \check{\lambda}_{n}^{(S \mathcal{R})^{2}} v^{*} \\
& B_{3}=\check{\lambda}_{n}^{(\mathcal{S R})^{2}} v^{*}-\check{\lambda}_{n}^{(\mathcal{S D})} \check{\lambda}_{n}^{(\mathcal{S R})^{2}}+2 \check{\lambda}_{n}^{(\mathcal{S D})} \check{\lambda}_{n}^{(\mathcal{S R})} v^{*} \\
& +\check{\lambda}_{n}^{(\mathcal{S R})^{2}} \lambda_{n}^{(\mathcal{R D})} v^{*} p_{n}^{(\mathcal{R})}+2 \check{\lambda}_{n}^{(\mathcal{S D})} \check{\lambda}_{n}^{(\mathcal{S R})} \lambda_{n}^{(\mathcal{R D})} v^{*} p_{n}^{(\mathcal{R})} \\
& B_{4}=\check{\lambda}_{n}^{(\mathcal{S D})} v^{*}-2 \check{\lambda}_{n}^{(\mathcal{S D})} \check{\lambda}_{n}^{(\mathcal{S R})}+2 \check{\lambda}_{n}^{(\mathcal{S R})} v^{*} \\
& +2 \check{\lambda}_{n}^{(\mathcal{S D})} \lambda_{n}^{(\mathcal{R D})} v^{*} p_{n}^{(\mathcal{R})}+3 \check{\lambda}_{n}^{(\mathcal{S R})} \lambda_{n}^{(\mathcal{R} D)} v^{*} p_{n}^{(\mathcal{R})}
\end{aligned}
$$

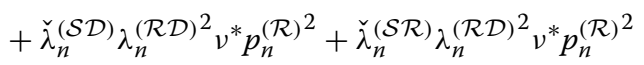

$$
\begin{aligned}
& -2 \check{\lambda}_{n}^{(\mathcal{S D})} \check{\lambda}_{n}^{(\mathcal{S R})} \lambda_{n}^{(\mathcal{R} \mathcal{D})} p_{n}^{(\mathcal{R})}
\end{aligned}
$$

$$
\begin{aligned}
B_{5} & =\frac{1}{2} \frac{B_{1}}{B_{2}}-\frac{1}{27} \frac{B_{3}^{3}}{B_{2}^{3}}+\frac{1}{6} \frac{B_{3} B_{4}}{B_{2}^{2}} \\
B_{6} & =\sqrt{B_{5}^{2}-\frac{\left(B_{3}^{2}-3 B_{2} B_{4}\right)^{3}}{729 B_{2}^{6}}} \\
\check{p}_{n}^{*(\mathcal{S})} & =\left[\left(B_{5}+B_{6}\right)^{\frac{1}{3}}+\left(B_{5}-B_{6}\right)^{\frac{1}{3}}-\frac{1}{3} \frac{B_{3}}{B_{2}}\right]^{+} .
\end{aligned}
$$

Similar to the water-filling solution, these solutions depend on the channel coefficients as well as on Lagrange multipliers $v$. Since channel coefficients are given, only optimal Lagrange multipliers $v^{*}$ are unknown. If the obtained solutions (56) and (63) are applied to individual

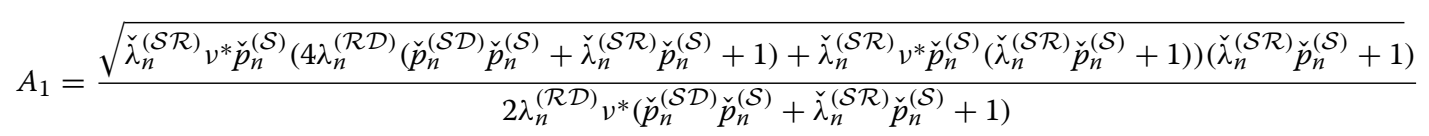


sum constraints (17) and (18), there is just a single equation with a single unknown variable $v^{*}$ left:

$$
\begin{array}{r}
\sum_{n \in \mathbb{N}}\left[A_{1}-A_{2}\right]^{+}-P_{\max }=0 \\
\sum_{n \in \mathbb{N}_{k}}\left[\left(B_{5}+B_{6}\right)^{\frac{1}{3}}+\left(B_{5}-B_{6}\right)^{\frac{1}{3}}-\frac{1}{3} \frac{B_{3}}{B_{2}}\right]^{+}-P_{\max }=0 .
\end{array}
$$

Finally, PAs $\check{\boldsymbol{p}}^{*((\mathcal{S}))}$, or $\boldsymbol{p}^{*(\mathcal{R})}$, can be obtained using a root finding algorithm, such as the bi-section method, to solve Equation (64), or (65), respectively.

\section{Non-adaptive decode and forward}

As proposed by Boostanimehr et al. in [28], the DF resource allocation problem can be reformulated such that the minimum function in objective (8) is replaced by two additional constraints. The objective $R^{\mathrm{DF}}$ of the equivalent allocation problem is given by

$$
\begin{array}{rlr}
R^{\mathrm{DF}} & =\sum_{n \in \mathbb{N}} \frac{1}{2} \frac{B}{N} \log _{2} 1+w_{n} & \\
\text { s.t. } \quad w_{n} \leq \check{p}_{n}^{(\mathcal{S})} \check{\lambda}_{n}^{(\mathcal{S D})}+p_{n}^{(\mathcal{R})} \lambda_{n}^{(\mathcal{R D})} & \forall n \in \mathbb{N} \\
& w_{n} \leq \check{p}_{n}^{(\mathcal{S})} \check{\lambda}_{n}^{(\mathcal{S R})} & \forall n \in \mathbb{N} .
\end{array}
$$

As objective (66) is concave and the corresponding constraint functions are affine, the separate power allocation problems (48) and (49) can be solved in an optimal way. For the resource allocation problem with the minimum function, this was not obvious.

To solve the problem, dual optimisation can be applied. The Lagrangian of the dual optimisation problem for the relay power constraint is given by

$$
\begin{aligned}
L= & R^{\mathrm{DF}}+\sum_{n \in \mathbb{N}}\left(v_{n}^{(\mathcal{R})} p_{n}^{(\mathcal{R})}\right) \\
& -\mu^{(\mathcal{R})}\left[\sum_{n \in \mathbb{N}} p_{n}^{(\mathcal{R})}-P_{\max }\right] \\
& -\sum_{n \in \mathbb{N}} \eta^{(\mathcal{R})}\left(w_{n}-\check{p}_{n}^{(\mathcal{S})} \check{\lambda}_{n}^{(\mathcal{S D})}-p_{n}^{(\mathcal{R})} \lambda_{n}^{(\mathcal{R D})}\right) \\
& -\sum_{n \in \mathbb{N}} \varrho^{(\mathcal{R})}\left(w_{n}-\check{p}_{n}^{(\mathcal{S})} \check{\lambda}_{n}^{(\mathcal{S R})}\right),
\end{aligned}
$$

where the different terms refer to the problem constraints. The solution can be obtained by applying and solving Karush-Kuhn-Tucker (KKT) conditions. This will not be done here in all details, but all cases that can occur in that process will be discussed shortly.

The main idea is to optimise the rate that is minimal for each subcarrier $n$. At the end, there will be three solutions corresponding to three subsets of $\mathbb{N}$ :

$$
\begin{array}{ll}
n \in \mathbb{C}_{\mathcal{R}}^{(\mathcal{R})} \subset \mathbb{N} & \forall \check{r}_{n}^{\mathcal{R}}<\check{r}_{n}^{\mathcal{D}} \\
n \in \mathbb{C}_{\mathcal{D}}^{(\mathcal{R})} \subset \mathbb{N} & \forall \check{r}_{n}^{\mathcal{R}}>\check{r}_{n}^{\mathcal{D}} \\
n \notin \mathbb{C}_{\mathcal{R}}^{(\mathcal{R})} \cup \mathbb{C}_{\mathcal{D}}^{(\mathcal{R})} & \forall \check{r}_{n}^{\mathcal{R}}=\check{r}_{n}^{\mathcal{D}} .
\end{array}
$$

If the achievable rate at the relay is crucial, the $n$th subcarrier is assigned to the first set $\mathbb{C}_{\mathcal{R}}^{(\mathcal{R})}$. If the rate at the destination node is crucial, $n$ is assigned to set $\mathbb{C}_{\mathcal{D}}$, or if the rates are identical, $n$ belongs to neither of these sets. The concrete conditions for subcarrier assignments to sets $\mathbb{C}_{\mathcal{R}}^{(\mathcal{R})}$ and $\mathbb{C}_{\mathcal{D}}^{(\mathcal{R})}$ can be derived later for each of the specific solutions individually.

First, optimisation can be done for the case $\check{r}_{n}^{\mathcal{R}}<\check{r}_{n}^{\mathcal{D}}$ that corresponds to Lagrange multipliers $\eta^{(\mathcal{R})}=0$ and $\varrho^{\mathcal{R}} \neq 0$. Here, objective (66) is always limited by constraint (68) as the third line in Lagrangian (69) vanishes. Variable $w_{n}$ corresponds to rate $\check{r}_{n}^{\mathcal{R}}$. As $\check{r}_{n}^{\mathcal{R}}$ does not depend on relay power $p_{n}^{(\mathcal{R})}$, any power spend to $p_{n}^{(\mathcal{R})}$ is wasted. It cannot increase the rate corresponding to subcarrier $n$. Such subcarriers should therefore not get any relay power at all:

$$
p_{n}^{(\mathcal{R})}=0 \quad \forall n \in \mathbb{C}_{\mathcal{R}}^{(\mathcal{R})} .
$$

Nevertheless, this solution is restricted to subcarriers in set $\mathbb{C}_{\mathcal{R}}^{(\mathcal{R})}$. If solution (73) is applied to definition (70), set $\mathbb{C}_{\mathcal{R}}^{(\mathcal{R})}$ is given by

$$
n \in \mathbb{C}_{\mathcal{R}}^{(\mathcal{R})} \quad \forall \check{\lambda}_{n}^{(\mathcal{S R})}<\check{\lambda}_{n}^{(\mathcal{S D})} .
$$

The found solution $p_{n}^{(\mathcal{R})}=0$ is equivalent to ignoring that subcarrier $n$ at the relay node. Relay $\mathcal{R}$ will not forward messages on the $n$th subcarrier, even if the $\mathrm{nDF}$ transmission scheme always require successful decoding at relay $\mathcal{R}$. The transmission rate therefore has to be reduced such that $\mathcal{R}$ is able to decode successfully on that subcarrier, even if $\mathcal{R}$ will not use that subcarrier for transmission. This seems to be a contradiction; however, it is the optimal behaviour in the $\mathrm{nDF}$ case. If $\check{\lambda}_{n}^{(\mathcal{S R})}<\check{p}_{n}^{(\mathcal{S D})}$, the transmission rate is adjusted to the $\mathcal{S} \rightarrow \mathcal{R}$ path and the destination node does not need any additional message from the indirect path to decode error free. Any relay power spend on forwarding over $\mathcal{R}$ is therefore wasted. In contrast, the solution of the 
aDF scheme will not suffer from this problem as aDF is able to switch to another relaying protocol in such a case.

Next, the case $\check{r}_{n}^{\mathcal{R}}>\check{r}_{n}^{\mathcal{D}}$ is considered that corresponds to $\eta^{(\mathcal{R})} \neq 0$ and $\varrho^{(\mathcal{R})}=0$. Since the fourth term in (69) is ignored, $w_{n}$ corresponds to rate $\breve{r}_{n}^{\mathcal{D}}$. Solving the KKT conditions will lead here to a modified water-filling solution:

$$
p_{n}^{(\mathcal{R})}=\left[\frac{1}{\mu^{(\mathcal{R})}}-\frac{1+\check{p}_{n}^{(\mathcal{S}) \check{\lambda}_{n}^{(\mathcal{S D})}}}{\lambda_{n}^{(\mathcal{R} \mathcal{D})}}\right]^{+} \quad \forall n \in \mathbb{C}_{\mathcal{D}}^{(\mathcal{R})}
$$

Set $\mathbb{C}_{\mathcal{D}}^{(\mathcal{R})}$ follows inserting (75) into the set definition $\check{r}_{n}^{\mathcal{R}}>\check{\check{r}}_{n}^{\mathcal{D}}$ :

$n \in \mathbb{C}_{\mathcal{D}}^{(\mathcal{R})} \quad \forall\left(\check{\lambda}_{n}^{(\mathcal{S R})}>\check{\lambda}_{n}^{(\mathcal{S D})}\right) \wedge\left(\mu^{(\mathcal{R})}>\frac{\lambda_{n}^{(\mathcal{R D})}}{\check{p}_{n}^{(\mathcal{S})} \check{\lambda}_{n}^{(\mathcal{S R})}+1}\right)$.

Finally, we have to consider the last case. If neither of the rates is greater than the other, both rates have to be equal $\check{r}_{n}^{\mathcal{R}}=\check{r}_{n}^{\mathcal{D}}$ in consequence.

If $\eta^{(\mathcal{R})} \neq 0$ and $\varrho^{(\mathcal{R})} \neq 0$, both conditions (67) and (68) have to be fulfilled simultaneously with equality. This leads to equation

$$
\check{p}_{n}^{(\mathcal{S})} \check{\lambda}_{n}^{(\mathcal{S R})}=\check{p}_{n}^{(\mathcal{S})} \check{\lambda}_{n}^{(\mathcal{S D})}+p_{n}^{(\mathcal{R})} \lambda_{n}^{(\mathcal{R} \mathcal{D})} .
$$

As source power $\check{p}_{n}^{(\mathcal{S})}$ is assumed to be known for separate optimisation, the optimal relay power $p_{n}^{(\mathcal{R})}$ can be computed directly:

$$
p_{n}^{(\mathcal{R})}=\frac{\check{\lambda}_{n}^{(\mathcal{S R})}-\check{\lambda}_{n}^{(\mathcal{S D})}}{\lambda_{n}^{(\mathcal{R} \mathcal{D})}} \check{p}_{n}^{(\mathcal{S})} \quad \forall n \notin \mathbb{C}_{\mathcal{R}}^{(\mathcal{R})} \cup \mathbb{C}_{\mathcal{D}}^{(\mathcal{R})}
$$

An interesting observation concerning solution (78) is that it is independent of Lagrange multiplier $\mu^{(\mathcal{R})}$. It therefore might not be possible to fulfil the sum constraint of the relay node. However, the allocation of a subcarrier to a sets $\mathbb{C}_{\mathcal{A}}^{(\mathcal{R})}$ depends on the choice of Lagrange multiplier $\mu^{(\mathcal{R})}$. Due to the first solution (73) where zero power is assigned, it will always be possible to fulfil the sum constraint with inequality, i.e. not all the available relay power is used.

If relay $\mathcal{R}$ moves close to destination $\mathcal{D}$, solution (77) will be used for most of the subcarriers. In that case, only little power is required to transmit the information over the $\mathcal{R} \rightarrow \mathcal{D}$ path, and in fact, any additional transmit power at relay $\mathcal{R}$ cannot improve the transmission rate any further. If relay $\mathcal{R}$ is close to the source node, all the available transmit power can be used to increase sum rate $R^{\mathrm{DF}}$.

Another important observation is that solution (78) is always non-negative. Since subcarriers $n \notin \mathbb{C}_{\mathcal{R}}^{(\mathcal{R})} \cup \mathbb{C}_{\mathcal{D}}^{(\mathcal{R})}$ are restricted to condition $\check{\lambda}_{n}^{(\mathcal{S R})} \geq \check{p}_{n}^{(\mathcal{S D})}$, the factor in front of source power $\breve{p}_{n}^{(\mathcal{S})}$ is ensured to be non-negative.

The final solution for relay power $p_{n}^{*(\mathcal{R})}$ is given by merging solutions (73), (75) and (78):

$$
p_{n}^{*(\mathcal{R})}= \begin{cases}0 & \forall n \in \mathbb{C}_{\mathcal{R}}^{(\mathcal{R})} \\ {\left[\frac{1}{\mu^{(\mathcal{R})}}-\frac{1+\check{p}_{n}^{(\mathcal{S})} \check{\lambda}_{n}^{(\mathcal{S D})}}{\lambda_{n}^{(\mathcal{R} \mathcal{D})}}\right]^{+} \quad \forall n \in \mathbb{C}_{\mathcal{D}}^{(\mathcal{R})}} \\ \frac{\check{\lambda}_{n}^{(\mathcal{S R})}-\check{\lambda}_{n}^{(\mathcal{S D})} \check{p}_{n}^{(\mathcal{S})}}{\lambda_{n}^{(\mathcal{R} \mathcal{D})}} & \forall n \notin \mathbb{C}_{\mathcal{R}}^{(\mathcal{R})} \cup \mathbb{C}_{\mathcal{D}}^{(\mathcal{R})}\end{cases}
$$

As in the AF case, unknown Lagrange multiplier $\mu^{(\mathcal{R})}$ or power $p_{n}^{*(\mathcal{R})}$, respectively, can be determined with the help of the bi-section method and the relay sum power constraint.

After having solved the relay power allocation problem, the source power problem can be tackled equivalently. There are again three solutions for the nDF source power problem (49) given by

$$
\check{p}_{n}^{*(\mathcal{S})}= \begin{cases}{\left[\frac{1}{\mu^{(\mathcal{S})}}-\frac{1}{\lambda_{n}^{\left(\mathcal{S}_{k} \mathcal{R}\right)}}\right]^{+}} & \forall n \in \mathbb{C}_{\mathcal{R}}^{(\mathcal{S})} \\ {\left[\frac{1}{\mu^{(\mathcal{S})}}-\frac{1+p_{n}^{(\mathcal{R})} \lambda_{n}^{(\mathcal{R} \mathcal{D})}}{\lambda_{n}^{\left(\mathcal{S}_{k} \mathcal{D}\right)}}\right]^{+}} & \forall n \in \mathbb{C}_{\mathcal{D}}^{(\mathcal{S})} \\ \frac{\lambda_{n}^{(\mathcal{R} D)}}{\lambda_{n}^{\left(\mathcal{S}_{k} \mathcal{R}\right)}-\lambda_{n}^{\left.(\mathcal{S})_{k} \mathcal{D}\right)} p_{n}^{(\mathcal{R})}} & \forall n \notin \mathbb{C}_{\mathcal{R}}^{(\mathcal{S})} \cup \mathbb{C}_{\mathcal{D}}^{(\mathcal{S})}\end{cases}
$$

where corresponding subsets are defined by

$$
\begin{aligned}
n \in \mathbb{C}_{\mathcal{R}}^{(\mathcal{S})} \quad & \forall\left(\lambda_{n}^{\left(\mathcal{S}_{k} \mathcal{R}\right)}<\lambda_{n}^{\left(\mathcal{S}_{k} \mathcal{D}\right)}\right) \\
& \vee\left(\mu^{(\mathcal{S})}>\frac{\lambda_{n}^{\left(\mathcal{S}_{k} \mathcal{D}\right)} \lambda_{n}^{\left(\mathcal{S}_{k} \mathcal{R}\right)}-\lambda_{n}^{\left(\mathcal{S}_{k} \mathcal{R}\right)^{2}}}{\lambda_{n}^{\left(\mathcal{S}_{k} \mathcal{D}\right)}-\lambda_{n}^{\left(\mathcal{S}_{k} \mathcal{R}\right)}-\lambda_{n}^{\left(\mathcal{S}_{k} \mathcal{R}\right)} \lambda_{n}^{(\mathcal{R})} p_{n}^{(\mathcal{R})}}\right)
\end{aligned}
$$

$$
\begin{aligned}
n \in \mathbb{C}_{\mathcal{D}}^{(\mathcal{S})} \quad & \forall\left(\lambda_{n}^{\left(\mathcal{S}_{k} \mathcal{R}\right)}>\lambda_{n}^{\left(\mathcal{S}_{k} \mathcal{D}\right)}\right) \\
& \wedge\left(\mu^{(\mathcal{S})}<\frac{\lambda_{n}^{\left(\mathcal{S}_{k} \mathcal{D}\right)} \lambda_{n}^{\left(\mathcal{S}_{k} \mathcal{R}\right)}-\lambda_{n}^{\left(\mathcal{S}_{k} \mathcal{D}\right)^{2}}}{\lambda_{n}^{\left(\mathcal{S}_{k} \mathcal{D}\right)}-\lambda_{n}^{\left(\mathcal{S}_{k} \mathcal{R}\right)}-\lambda_{n}^{\left(\mathcal{S}_{k} \mathcal{R}\right)} \lambda_{n}^{(\mathcal{R D})} p_{n}^{(\mathcal{R})}}\right) .
\end{aligned}
$$

Another aspect that should be discussed here concerns the assignment of subcarriers to the sets $\mathbb{C}^{(\mathcal{R})}$ and $\mathbb{C}^{(\mathcal{S})}$. 
Intuitively, it seems reasonable to choose equivalent sets $\mathbb{C}_{\mathcal{R}}^{(\mathcal{R})}=\mathbb{C}_{\mathcal{R}}^{(\mathcal{S})}$ and $\mathbb{C}_{\mathcal{D}}^{(\mathcal{R})}=\mathbb{C}_{\mathcal{D}}^{(\mathcal{S})}$ because they correspond to the same rate constraints $\check{r}_{n}^{\mathcal{R}}<\check{r}_{n}^{\mathcal{D}}$ or $\check{r}_{n}^{\mathcal{R}}>\check{r}_{n}^{\mathcal{D}}$. However, the allocation to sets $\mathbb{C}^{(\mathcal{R})}$ and $\mathbb{C}^{(\mathcal{S})}$ can be different in the iterative PA process. Actually, this is reasonable as each optimisation step corresponds to an improvement of the worst rate. In a following step, the other rate in the minimum function might become worse and the subcarrier assignments can change.

Contrary, if the iterative process converges to a specific solution $\check{\boldsymbol{p}}^{(\mathcal{S})}, \boldsymbol{p}^{(\mathcal{R})}$, the sets are identical and will not change any further. (If a specific solution has been found, the relation between rates $\check{r}_{n}^{\mathcal{R}}$ and $\check{r}_{n}^{\mathcal{D}}$ will also not change any further and the sets are fixed.)

\section{Adaptive decode and forward}

The adaptive DF scheme is similar to the non-adaptive scheme, but the relay is no longer required to decode without errors. If decoding is not successful at the relay node, it will not forward any message on that subcarrier and power optimisation can be done according to the direct link. The aDF solution can be obtained by modifying the nDF solutions. The transmission rate $\breve{r}_{n}^{\mathrm{aDF}}$ is only limited by the rate $\check{r}_{n}^{\text {Dir }}$ if $\check{\lambda}_{n}^{(\mathcal{S R})}<\check{\lambda}_{n}^{(\mathcal{S D})}$. In that case, the relay does not forward on that subcarrier, and the waterfilling solution is used to allocate the transmit power of the source nodes:

$$
\begin{aligned}
& p_{n}^{*(\mathcal{R})}=0 \\
& \forall n \in \mathbb{N}, \check{\lambda}_{n}^{(\mathcal{S D})}>\check{\lambda}_{n}^{(\mathcal{S R})} \\
& \check{p}_{n}^{*(\mathcal{S})}=\left[\frac{1}{\mu^{(\mathcal{R})}}-\frac{1}{\check{\lambda}_{n}^{(\mathcal{S D})}}\right]^{+} \forall n \in \mathbb{N}, \check{\lambda}_{n}^{(\mathcal{S D})}>\check{\lambda}_{n}^{(\mathcal{S R})} .
\end{aligned}
$$

On the other hand, if $\check{\lambda}_{n}^{(\mathcal{S R})}>\check{\lambda}_{n}^{(\mathcal{S D})}$, the solution of the nDF in Equations (79) and (81) can be used because rate $\check{r}_{n}^{\mathrm{nDF}}$ is always better than the rate $\check{r}_{n}^{\mathrm{Dir}}$ of the direct path.

\section{Compress and forward}

The solution for the CF power allocation problem can be derived equivalently to the AF case. There is just a slightly changed objective due to the changed SNR in the rate function.

For the relay node, the optimal power allocation of the separate CF problem is given by
Solving the source power problem leads to several solutions, where again just a single solution

$$
\check{p}_{n}^{*(\mathcal{S})}=\left[\left(C_{5}-C_{6}\right)^{\frac{1}{3}}+\left(C_{5}+C_{6}\right)^{\frac{1}{3}}+\frac{1}{3} \frac{C_{3}}{C_{2}}\right]^{+}
$$

is feasible. Variables $C_{1}$ to $C_{6}$ are given by

$$
\begin{aligned}
& C_{1}=\check{\lambda}_{n}^{(\mathcal{S D})}-v^{*}+\check{\lambda}_{n}^{(\mathcal{S D})} \lambda_{n}^{(\mathcal{R D})} p_{n}^{(\mathcal{R})} \\
& +\check{\lambda}_{n}^{(\mathcal{S R})} \lambda_{n}^{(\mathcal{R} \mathcal{D})} p_{n}^{(\mathcal{R})}-\lambda_{n}^{(\mathcal{R D})} v^{*} p_{n}^{(\mathcal{R})} \\
& C_{2}=\check{\lambda}_{n}^{(\mathcal{S D})^{3}} v^{*}+\check{\lambda}_{n}^{(\mathcal{S D})} \check{\lambda}_{n}^{(\mathcal{S R})^{2}} v^{*}+2 \check{\lambda}_{n}^{(\mathcal{S D})^{2}} \check{\lambda}_{n}^{(\mathcal{S R})} v^{*} \\
& +\check{\lambda}_{n}^{(\mathcal{S D})^{3}} \lambda_{n}^{(\mathcal{R D})} v^{*} p_{n}^{(\mathcal{R})}+\check{\lambda}_{n}^{(\mathcal{S D})^{2}} \check{\lambda}_{n}^{(\mathcal{S R})} \lambda_{n}^{(\mathcal{R D})} v^{*} p_{n}^{(\mathcal{R})}
\end{aligned}
$$

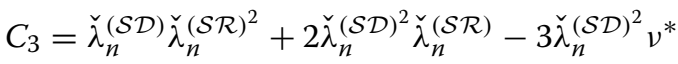

$$
\begin{aligned}
& -\check{\lambda}_{n}^{(\mathcal{S R})^{2}} \nu^{*}+\check{\lambda}_{n}^{(\mathcal{S D})^{3}}-4 \check{\lambda}_{n}^{(\mathcal{S D})} \check{\lambda}_{n}^{(\mathcal{S R})} v^{*} \\
& +\check{\lambda}_{n}^{(\mathcal{S D})^{3}} \lambda_{n}^{(\mathcal{R D})} p_{n}^{(\mathcal{R})}+\check{\lambda}_{n}^{(\mathcal{S D})^{2}} \check{\lambda}_{n}^{(\mathcal{S R})} \lambda_{n}^{(\mathcal{R D})} p_{n}^{(\mathcal{R})} \\
& -3 \check{\lambda}_{n}^{(\mathcal{S D})^{2}} \lambda_{n}^{(\mathcal{R} \mathcal{D})} \nu^{*} p_{n}^{(\mathcal{R})}-2 \check{\lambda}_{n}^{(\mathcal{S D})} \check{\lambda}_{n}^{(\mathcal{S R})} \lambda_{n}^{(\mathcal{R D})} \nu^{*} p_{n}^{(\mathcal{R})}
\end{aligned}
$$

$$
\begin{aligned}
& C_{4}=2 \check{\lambda}_{n}^{(\mathcal{S D})} \check{\lambda}_{n}^{(\mathcal{S R})}-3 \check{\check{\lambda}}_{n}^{(\mathcal{S D})} \nu^{*}-2 \check{\lambda}_{n}^{(\mathcal{S R})} v^{*} \\
& +2 \check{\lambda}_{n}^{(\mathcal{S D})^{2}} \lambda_{n}^{(\mathcal{R D})} p_{n}^{(\mathcal{R})}-3 \check{\lambda}_{n}^{(\mathcal{S D})} \lambda_{n}^{(\mathcal{R D})} \nu^{*} p_{n}^{(\mathcal{R})} \\
& +2 \check{\lambda}_{n}^{(\mathcal{S D})^{2}}-\check{\lambda}_{n}^{(\mathcal{S R})} \lambda_{n}^{(\mathcal{R} \mathcal{D})} \nu^{*} p_{n}^{(\mathcal{R})} \\
& +2 \check{\lambda}_{n}^{(\mathcal{S D})} \check{\lambda}_{n}^{(\mathcal{S R})} \lambda_{n}^{(\mathcal{R} \mathcal{D})} p_{n}^{(\mathcal{R})} \\
& C_{5}=\frac{1}{2} \frac{C_{1}}{C_{2}}+\frac{1}{27} \frac{C_{3}^{3}}{C_{2}^{3}}+\frac{1}{6} \frac{C_{3} C_{4}}{C_{2}^{2}} \\
& C_{6}=\sqrt{C_{5}^{2}-\frac{\left(C_{3}^{2}+3 C_{2} C_{4}\right)^{3}}{729 C_{2}{ }^{6}}} .
\end{aligned}
$$

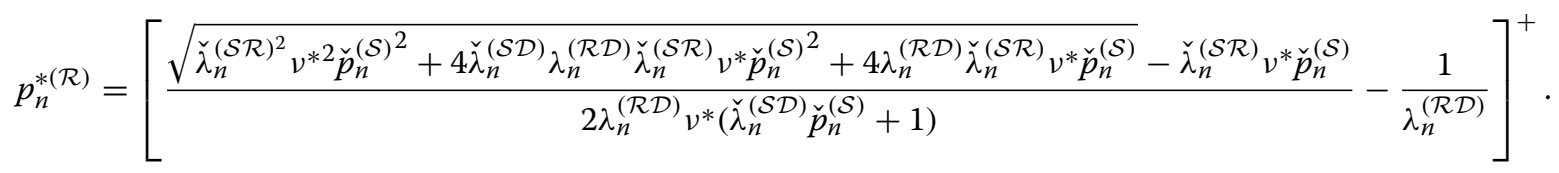




\section{Abbreviations}

aDF: Adaptive decode and forward; AF: Amplify and forward; CA: Carrier allocation; CF: Compress and forward; CSI: Channel state information; DF: Decode and forward; JA: Joint allocation; KKT: Karush-Kuhn-Tucker; MARC: Multiple-access relay channel; nDF: Non-adaptive decode and forward; OFDM: Orthogonal frequency division multiplexing; OFDMA: Orthogonal frequency division multiple access; PA: Power allocation; SNR: Signal-to-noise ratio.

\section{Competing interests}

The authors declare that they have no competing interests.

\section{Received: 31 May 2012 Accepted: 7 June 2013}

\section{Published: 24 June 2013}

\section{References}

1. E van der Meulen, Three-terminal communication channels. Adv. Appl. Probability. 3, 120-154 (1971)

2. E van der Meulen, Transmission of information in a T-terminal discrete memoryless channel. PhD dissertation, University of California, 1968

3. T Cover, A Gamal, Capacity theorems for the relay channel. IEEE Trans. Inf. Theory. 25(5), 572-584 (1979)

4. G Kramer, A van Wijngaarden, in Proceedings of the IEEE International Symposium on Information Theory (ISIT) 2000. On the white Gaussian multiple-access relay channel (Sorrento, 25-30 June 2000), p. 40

5. J Laneman, D Tse, G Wornell, Cooperative diversity in wireless networks: efficient protocols and outage behavior. IEEE Trans. Inf. Theory. 50(12), 3062-3080 (2004)

6. L Sankaranarayanan, G Kramer, N Mandayam, in Proceedings of the 1st Annual IEEE Communications Society Conference on Sensor and Ad HoC Communications and Networks (SECON) 2004. Hierarchical sensor networks: capacity bounds and cooperative strategies using the multiple-access relay channel model (Santa Clara, 4-7 Oct 2004), pp. 191-199

7. A Høst-Madsen, J Zhang, Capacity bounds and power allocation for wireless relay channels. IEEE Trans, Inf. Theory. 51(6), 2020-2040 (2005)

8. G Kramer, M Gastpar, P Gupta, Cooperative strategies and capacity theorems for relay networks. IEEE Trans, Inf. Theory. 51(9), 3037-3063 (2005)

9. A Goldsmith, J Jiang, S Cui, in Proceedings of the IEEE International Symposium on Information Theory (ISIT) 2009. Achievable rates and capacity for Gaussian relay channels with correlated noises (Seoul, 28 June), pp. 179-183

10. V Kühn V, Wireless Communications over MIMO Channels: Applications to CDMA and Multiple Antenna Systems. (Wiley, Chichester, 2006)

11. CWong, R Cheng, K Lataief, R Murch, Multiuser OFDM with adaptive subcarrier, bit, and power allocation. IEEE J. Sel. Areas Commun. 17(10), 1747-1758 (1999)

12. CWong, Y Tsui, RS Cheng, KB Letaief, A real-time sub-carrier allocation scheme for multiple access downlink OFDM transmission, vol. 2, (Amsterdam, 19-22 Sept 1999), pp. 1124-1128

13. H Yin, H Liu, in Proceedings of the IEEE Global Telecommunications Conference (GLOBECOM) 2000, vol. 1. An efficient multiuser loading algorithm for OFDM-based broadband wireless systems (San Francisco, 27 Nov-1 Dec 2000), pp. 103-107

14. W Rhee, J Cioffi, in Proceedings of the 51st IEEE Vehicular Technology Conference (VTC-Spring) 2000, vol. 2. Increase in capacity of multiuser OFDM system using dynamic subchannel allocation (Tokyo, 15-18 May 2000), pp. 1085-1089

15. N Ermolova, B Makarevitch, in Proceedings of the 16th IEEE International Symposium on Personal, Indoor and Mobile Radio Communications (PIMRC) 2005, vol. 1. Power and subcarrier allocation algorithms for OFDMA systems (Berlin, 11-14 Sept 2005), pp. 352-356

16. K Seong, M Mohseni, J Cioffi, in Proceedings of the IEEE International Symposium on Information Theory (ISIT) 2006. Optimal resource allocation for OFDMA downlink systems (Seattle, 9-14 July 2006), pp. 1394-1398

17. W Yu, R Lui, Dual methods for nonconvex spectrum optimization of multicarrier systems. IEEE Trans. Commun. 54(7), 1310-1322 (2006)

18. I Wong, B Evans, in Proceedings of the IEEE International Conference on Acoustics, Speech and Signal Processing (ICASSP) 2007, vol. 3. Optimal OFDMA resource allocation with linear complexity to maximize ergodic weighted sum capacity (Honolulu, 15), pp. III-601-|II-604
19. G Yu, Z Zhang, Y Chen, S Chen, P Qiu, in Proceedings of the International Conference on Wireless Communications, Networking and Mobile Computing (WCNM) 2005, vol. 1. Power allocation for non-regenerative OFDM relaying channels (Wuhan, 23-26 Sept 2005), pp. 185-188

20. Y Wang, X Qu, TWu, B Liu, in Proceedings of the 65th IEEE Vehicular Technology Conference (VTC-Spring) 2007. Power allocation and subcarrier pairing algorithm for regenerative OFDM relay system (Dublin, 22-25 Apr 2007), pp. $2727-2731$

21. I Hammerström, A Wittneben, in Proceedings of the IEEE International Conference on Communications (ICC) 2006, vol. 10. On the optimal power allocation for nonregenerative OFDM relay links (Istanbul, 11-15 June 2006), pp. 4463-4468

22. I Hammerström, A Wittneben, Power allocation schemes for amplify-and-forward MIMO-OFDM relay links. IEEE Trans. Wireless Commun. 6(8), 2798-2802 (2007)

23. S Zhang, $V$ Lau, Resource allocation for OFDMA system with orthogonal relay using rateless code. IEEE Trans. Wireless Commun. 7(11), 4534-4540 (2008)

24. L Vandendorpe, J Louveaux, O Oguz, A Zaidi, in Proceedings of the 3rd International Symposium on Wireless Pervasive Computing (ISWPC) 2008. Improved OFDM transmission with DF relaying and power allocation for a sum power constraint (Santorini, 7-9 May 2008), pp. 665-669

25. L Vandendorpe, R Durán, J Louveaux, A Zaidi, in Proceedings of the IEEE International Conference on Communications (ICC) 2008. Power allocation for OFDM transmission with DF relaying (Beijing, 19-23 May 2008), pp. 3795-3800

26. Y Li, W Wang, J Kong, W Hong, X Zhang, M Peng, in Proceedings of the IEEE International Conference on Communications (ICC) 2008. Power allocation and subcarrier pairing in OFDM-based relaying networks (Beijing, 19-23 May 2008), pp. 2602-2606

27. L Vandendorpe, J Louveaux, O Oguz, A Zaidi, in Proceedings of the IEEE International Conference on Communications (ICC) 2009. Power allocation for improved DF relayed OFDM transmission: the individual power constraint case (Dresden, 14-18 June 2009), pp. 1-6

28. H Boostanimehr, O Duval, V Bhargava, F Gagnon, in Proceedings of the IEEE International Conference on Communications (ICC) 2010. Selective subcarrier pairing and power allocation for decode-and-forward OFDM relay systems (Cape Town, 23-27 May 2010), pp. 1-5

29. F Shi, C Zhao, in Proceedings of the 6th International Conference on Wireless Communications Networking and Mobile Computing (WiCOM) 2010. A low complexity power allocation approach for OFDM systems with DF relay (Chengdu, 23-25 Sept 2010), pp. 1-5

30. C CHsu, H Su, P Lin, Joint subcarrier pairing power allocation for OFDM transmission with decode-and-forward relaying. IEEE Trans. Signal Process. 59, 399-414 (2011)

31. T Ng, W Yu, Joint optimization of relay strategies and resource allocations in cooperative cellular networks. IEEE J. Sel. Areas Commun. 25(2), 328-339 (2007)

32. K Jitvanichphaibool, R Zhang, Y Liang, Optimal resource allocation for two-way relay-assisted OFDMA. IEEE Trans. Veh. Technol. 58(7), 3311-3321 (2009)

33. M El Soussi, A Zaidi, J Louveaux, L Vandendorpe, in Proceedings of the 4th International Symposium on Communications, Control and Signal Processing (ISCCSP) 2010. Sum-rate optimized power allocation for the OFDM multiple access relay channel (Limassol, 3-5 Mar 2010), pp. 1-6

34. C Shannon, A mathematical theory of communication. Bell Syst Tech. J. 27, 379-423 (1948)

35. S Schedler, V Kuehn, in Proceedings of the 9th International ITG Conference on Systems, Communications and Coding 2013 (SCC'2013). Distributed resource allocation for the MARC with amplify and forward and partial CSI (Munich, 21-24Jan 2013)

36. S Boyd, L Vandenberghe, Convex Optimization. (Cambridge University Press, New York, 2004)

37. S Boyd, L Vandenberghe, Subgradients. (Notes for EE364b, Stanford University, Winter 2006-07, 2011). http://www.stanford.edu/class/ ee364b/notes/subgradients_notes.pdf. Accessed 30 Mar 2012

38. S Boyd, A Mutapcic, Subgradient methods. (Notes for EE364b, Stanford University, Winter 2006-07, 2007). http://www.stanford.edu/class/ ee364b/notes/subgrad_method_notes.pdf. Accessed 30 Mar 2012

39. H Kuhn, The Hungarian method for the assignment problem. Nava Res. Logistics Q. 2(1-2), 83-97 (1955) 
40. GA Mills-Tettey, A Stentz, MB Dias, The Dynamic Hungarian Algorithm for the Assignment Problem with Changing Costs, vol. 1. (Robotics Institute, Carnegie Mellon University, Pittsburgh, 2007), pp. 1-13

41. T Rappaport, Wireless Communications: Principles and Practice, 2nd edn. (Upper Saddle River, Prentice Hall, 2001)

42. H Zhang, Y Liu, M Tao, Resource allocation with subcarrier pairing in OFDMA two-way relay networks. IEEE Wireless Commun Lett. 1(2), 61-64 (2012)

43. S Schedler, A Angierski, V Kuehn, in Proceedings of the IEEE Wireless Communications and Networking Conference (WCNC) 2012. Resource allocation for the AF multiple access relay channel with OFDMA (Paris, 1-4 April 2012)

doi:10.1186/1687-6180-2013-120

Cite this article as: Schedler and Kühn: Resource allocation for the multipleaccess relay channels and OFDMA. EURASIP Journal on Advances in Signal Processing 2013 2013:120.

\section{Submit your manuscript to a SpringerOpen ${ }^{\mathcal{O}}$ journal and benefit from:}

- Convenient online submission

- Rigorous peer review

- Immediate publication on acceptance

- Open access: articles freely available online

- High visibility within the field

- Retaining the copyright to your article

Submit your next manuscript at $\gg$ springeropen.com 